\title{
Reverse Mechanism Design
}

\author{
Nima Haghpanah \\ Northwestern University \\ EECS Department \\ nima.haghpanah@gmail.com
}

\author{
Jason Hartline \\ Northwestern University \\ EECS Department \\ hartline@northwestern. edu
}

July 15, 2014

\begin{abstract}
Myerson's 1981 characterization of revenue-optimal auctions for single-dimensional agents follows from an amortized analysis of the incentives of a single agent. To optimize revenue in expectation, he maps values to virtual values which account for expected revenue gain but can be optimized pointwise. For single-dimensional agents the appropriate virtual values are unique and their closed form can be easily derived from revenue equivalence. A main challenge of generalizing the Myersonian approach to multi-dimensional agents is that the right amortization is not pinned down by revenue equivalence.

For multi-dimensional agents, the optimal mechanism may be very complex. Complex mechanisms are impractical and rarely employed. We give a framework for reverse mechanism design. Instead of solving for the optimal mechanism in general, we assume a (natural) specific form of the mechanism. As an example of the framework, for agents with unit-demand preferences, we restrict attention to mechanisms that sell each agent her favorite item or nothing. From this restricted form, we will derive multi-dimensional virtual values. These virtual values prove this form of mechanism is optimal for a large class of item-symmetric distributions over types. As another example of our framework, for bidders with additive preferences, we derive conditions for the optimality of posting a single price for the grand bundle.
\end{abstract}




\section{Introduction}

Optimal mechanisms for agents with multi-dimensional preferences are generally complex. This complexity makes them challenging to solve for and impractical to run. In a typical mechanism design approach, a model is posited and then the optimal mechanism is designed for the model. Successful mechanism design gives mechanisms that one could at least imagine running. By this measure, multi-dimensional mechanism design has had only limited success. In this paper we take the opposite approach, which we term reverse mechanism design. We start by imagining a restriction on mechanisms that would make them simple and reasonable to run, then we solve for sufficient conditions for a restricted mechanism to be optimal (among all mechanisms). Our approach is successful if the conditions under which restricted mechanisms are optimal are broad and representative of relevant settings.

This paper has two main contributions. The first is in codifying the method of virtual values from single-dimensional auction theory and extending it to agents with multi-dimensional preferences. The second is in applying this method to two paradigmatic classes of multi-dimensional preferences. The first class is unit-demand preferences (e.g., a homebuyer who wishes to buy at most one house); for this class we give sufficient conditions under which the posting a uniform price for all items is optimal. This result generalizes one of Alaei et al. (2013) for a consumer with values uniform on interval [0,1], and contrasts with an example of Thanassoulis (2004) for a consumer with values uniform on interval $[5,6]$ where uniform pricing is not optimal. The second class is additive preferences, for this class we give sufficient conditions under which the posting a price for the grand bundle is optimal. This result generalizes a recent result of Hart and Nisan $(2013)$ and relates to work of Armstrong (1999). Similarly to an approach of Alaei et al. (2013), these results for single-agent pricing problems described above can be generalized naturally to multi-agent auction problems.

Myerson's (1981) characterization of revenue optimal auctions for single-dimensional agents is the cornerstone of modern auction theory and mechanism design. This characterization is successful in describing simple and practical mechanisms in simple environments where the agents preferences are independent and identically distributed according to a well-behaved distribution. In this case, the optimal auction is reserve price based. Myerson's characterization is also successful in describing the complex optimal mechanism for agents with preferences that are non-identically distributed or distributed according to an ill-behaved distribution. However, due to this complexity, the resulting mechanism has limited application. The consequence of our work is similar in that we characterize simple optimal mechanisms for well-behaved preferences; but distinct in that it does not characterize optimal mechanisms beyond the class of well-behave preferences.

Myerson's approach is based on mapping agent values to appropriately defined virtual values and then optimizing the virtual surplus, i.e., the sum of the virtual values of agents served. Importantly, this approach replaces the global objective optimizing revenue in expectation over the distribution of agent values to the pointwise objective of optimizing virtual surplus on each profile of agent values. Furthermore, virtual surplus maximization leads to a simple and practical optimal mechanism in many environments. The simplicity of analysis by virtual values and of mechanisms resulting from optimizing virtual values has lead to a rich theory single-dimensional auction theory. Our multidimensional virtual values similarly give a pointwise objective and their optimization results in simple optimal mechanisms.

In the remainder of this section we review virtual values in mechanism design for singledimensional agents, describe the challenges in identifying virtual values for multi-dimensional pref- 
erences, and describe how restricting the form of the optimal mechanism make it possible to solve for multi-dimensional virtual values. To keep the exposition simple, we focus on designing mechanisms for a single agent.

Optimal Single-dimensional Mechanisms for Revenue. Revenue is a challenging objective for mechanism design because, due to the agents' incentives, it cannot be optimized pointwise. To illustrate this fact, consider a single agent wishing to buy a single item and whose value for the item is drawn uniformly from the $[0,1]$ interval. If we post a take-it-or-leave-it price of 0.3 then the agent will buy when his value is greater than 0.3 and pay 0.3 ; if we post a price of 0.5 the agent will buy when his value is greater than 0.5 and pay 0.5 . If our agent has value 0.3 then the first mechanism has the best possible revenue and the second mechanism has an inferior revenue of zero. If our agent has value 0.5 then the second mechanism has the best possible revenue of 0.5 and the first mechanism has an inferior revenue of 0.3 . While we cannot rank these mechanisms by revenue pointwise, i.e., for all values the agent might possess, in expectation over the distribution from which the agent's value is drawn, the mechanisms can be ranked and revenue tradeoffs across possible agent values can be optimized. With a uniform value on $[0,1]$, the agent buys in the first mechanism at a price of 0.3 with probability 0.7 for an expected revenue of 0.21 , and in the second mechanism at a price of 0.5 with probability 0.5 for an expected revenue of 0.25 .

Myerson (1981) solved for revenue optimal auctions for agents with single-dimensional preferences. He gives a definition of virtual values, claims that optimization of virtual surplus gives the optimal auction, and proves this claim by utilizing two properties of the virtual values. The following two properties collectively imply that the mechanism that optimizes virtual values is indeed the optimal mechanism 11 A virtual value function maps values pointwise to virtual values satisfying two properties:

- The pointwise optimization of virtual values gives an allocation rule that is incentive compatible. That is, there exist payments for this allocation rule that induce an agent to truthfully report his value.

- The virtual values are an amortization of the revenue. That is, the expected sum of the virtual values of the winners of any incentive compatible mechanism is equal to the expected revenue of that mechanism.

This definition of virtual value functions gives a roadmap to identifying the optimal mechanism: find a virtual value function (that satisfies the two conditions) and run the mechanism that maximizes virtual value pointwise. The identification of a virtual value function reduces the problem of optimization of the expected revenue (a global quantity) to the optimization of virtual surplus (a pointwise quantity).

In our single-dimensional example of the uniform distribution above, the appropriate virtual value function is $2 v-1$. Optimizing virtual value pointwise (for a single agent) means serving the agent if his virtual value is positive and not serving him if his value is negative. For this virtual value function, the agent will be served if his value is least 0.5 . This virtual value function satisfies the incentive compatibility condition: serving the agent if her value is above 0.5 is incentive

\footnotetext{
${ }^{1}$ Subsequently, in Section 3. we will define a more permissive version of the amortization property which corresponds to the ironed virtual values of Myerson (1981). The simpler definition here will, nonetheless, be sufficient for our introductory discussion.
} 
compatible and the appropriate payment is 0.5 if the agent is served and zero otherwise. This virtual value function satisfies the amortization condition: for posting any price $p$, the expected virtual value of the agent served and the expected revenue are equal. The former can be calculated by $\int_{p}^{1}(2 v-1) d v=p(1-p)$ and the latter was calculated as above as $p(1-p){ }^{2}$

The Challenge of Multi-dimensional Preferences. As described above, if a virtual value function that satisfies properties the incentive compatibility and amortization conditions can be identified then the optimal mechanism design problem is solved. For agents with single-dimensional preferences the function that satisfies the amortization property is unique and can be derived by a simple exercise. Omitting the details: uniqueness follows because there is only one path on the line between any value and the origin (and can be found via integration by parts). It then remains to check the incentive compatibility property, i.e., that pointwise optimization of virtual value is incentive compatible; by standard characterizations of incentive compatible mechanisms, this is a simple task as well. Multi-dimensional preferences are challenging because the amortization property does not uniquely pin down the virtual value function. Omitting the details: non-uniqueness follows because from any point in a multi-dimensional space there are many paths between the point and the origin. This difficulty has prevented the design of mechanisms for multi-dimensional agents that follows the virtual-value-based approach.

Multi-dimensional Virtual Values. To resolve the non-uniqueness of functions that satisfy the amortization property we consider additional constraints that the optimality of a restricted mechanism would place on virtual values.

We walk through this approach for the example of a unit-demand agent and the restriction to mechanisms that post a uniform price for each item. On one hand, under uniform pricing, the agent will always choose to buy his favorite item, or no item if all values are below the price. On the other hand, a mechanism that optimizes a multi-dimensional virtual value (for a unit-demand agent) would serve the agent the item he has the highest positive virtual value for, or no item if all virtual values are negative. Synthesizing these constraints, the following conditions are sufficient for virtual surplus maximization to imply optimality of uniform pricing.

- The virtual value function is a single-dimensional projection if the virtual value for the favorite item corresponds to the single-dimensional virtual value for the distribution of the value for the favorite item (the distribution of the maximum value) $]^{3}$

- The virtual value function is consistent with uniform pricing if there is a price such that (a) when the value for the favorite item exceeds the price the virtual value for the favorite item is non-negative and at least the virtual value of any other items and (b) when the value for the favorite item is below the price both virtual values are non-positive.

Any virtual value function that satisfies the consistency-with-uniform-pricing conditions can be easily seen to satisfy the incentive compatibility requirement. Thus, the identification of a virtual value function becomes one of simultaneously resolving the three conditions of amortization,

\footnotetext{
${ }^{2}$ Further discussion omitted, this amortized equivalence continues to hold for randomized mechanisms.

${ }^{3}$ Rationale: The mechanism has effectively projected the agent's multi-dimensional preference onto a single dimension. In this single dimension the unique function that satisfies the amortization property is the one given by the single-dimensional virtual values of Myerson (1981)
} 
single-dimensional projection, and consistency with uniform pricing (which implies incentive compatibility). Conditions on the distributions over values for that guarantee the existence of such a virtual value function are sufficient for the optimality of uniform pricing.

For two-dimensional preferences the amortization and single-dimensional-projection restrictions pin down a two-dimensional virtual value function uniquely. Specifically, as the virtual value for the favorite item is fixed by the restriction, only the virtual value for the other item must be determined. Essentially, we are left with a single-dimensional problem and in a single dimension the function that satisfies the amortization condition are unique. Our task is then to give sufficient conditions under which this unique virtual value function is consistent with uniform pricing. For higher-dimensional preferences, virtual value functions for the non-favorite items are not pinned down. Instead of deriving a formula for them, we generalize the sufficient condition from the twodimensional case and prove that there exists such virtual value function that satisfied all three constraints.

The restriction of selling a unit-demand agent his favorite item provides our main example of the framework of reverse mechanism design. We will also apply the framework to agents with additive preferences and give sufficient conditions for pricing the grand bundle to be optimal. The techniques we develop can be similarly applied to other environments and appropriate restrictions, and these extensions are an important topic for future work.

Sufficient Conditions for Optimality of Simple Mechanisms. The opening example of this introduction of selling one of many houses to a homebuyer with value distributed independently, identically, and uniformly from $[0,1]$ satisfies the conditions for optimality of the restriction to selling him only his favorite item. More generally, our framework identifies sufficient conditions for the distribution over agent values. Importantly these conditions allow for positive correlation between the agent's value for distinct items, a.k.a, affiliation. Such correlation is natural when values correlate, e.g., with initial wealth. Below we state the sufficient conditions for optimality of uniform pricing for unit-demand agents and grand-bundle pricing for additive agents for the special case of two items; the general multi-item statements are deferred to later in the paper.

A single-agent problem with unit-demand preferences is defined with a density function $f$ on a bounded set of types normalized to $T=[0,1]^{2}$, where $\left(t_{1}, t_{2}\right)$ is the pair of values for the two items. We consider only symmetric distributions and therefore only define $f$ on the subset of $T$ satisfying $t_{1} \geq t_{2}$, that is, where the first item is the favorite item. A uniform posted price mechanism posts a uniform price for each item, and lets the agent choose one of the two items or nothing based on his type. It is easiest to state the result in the following max-ratio representation of the distribution. The max-ratio representation of a density function $f$ is the function $\bar{f}$ that is defined by

$$
f\left(t_{1}, t_{2}\right)=\bar{f}\left(t_{1}, t_{2} / t_{1}\right) .
$$

The following theorem states that uniform pricing is optimal if $\bar{f}$ satisfies a supermodularity condition. Intuitively, this supermodularity implies that a higher value of $t_{1}$ signals a higher value of $t_{2} / t_{1}$ (see Figure 1).

Theorem 1. Posting a uniform price for each item is optimal if the max-ratio representation of the density function is log-supermodular, that is,

$$
\bar{f}\left(t_{1}, \theta\right) \times \bar{f}\left(t_{1}, \theta^{\prime}\right) \leq \bar{f}\left(t_{1}, \theta^{\prime}\right) \times \bar{f}\left(t_{1}^{\prime}, \theta\right), \quad t_{1} \leq t_{1}^{\prime}, \theta \geq \theta^{\prime} .
$$




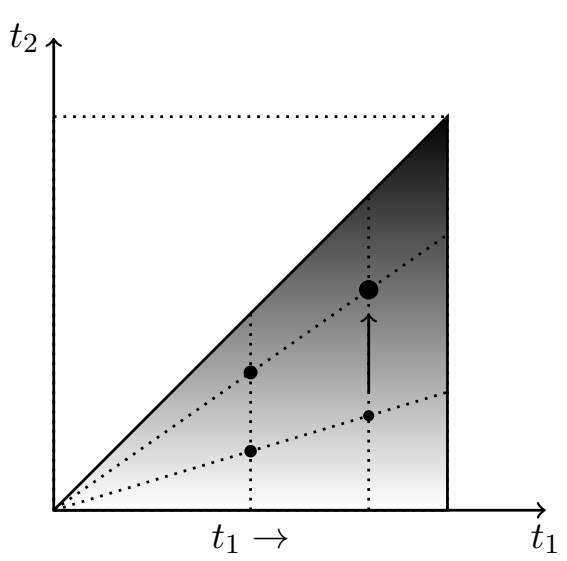

(a)

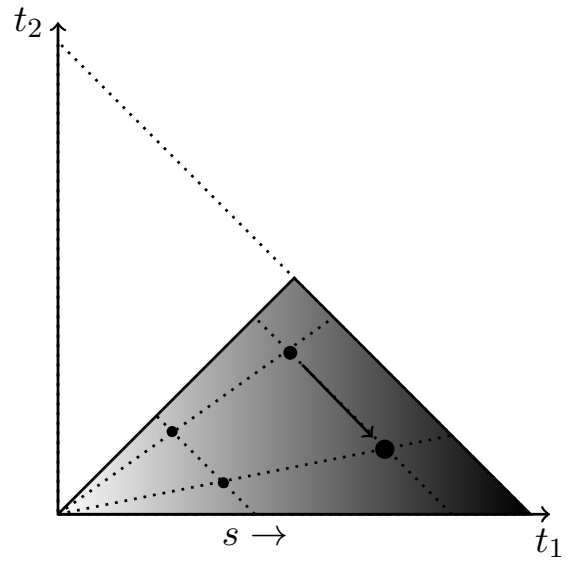

(b)

Figure 1: a) A distribution satisfying the supermodularity condition of Theorem 1. Dark color indicates relatively high mass. Roughly speaking, it states that the probability mass shifts upwards as the value for the favorite item, $t_{1}$, increases. b) A distribution satisfying the submodularity condition of Theorem 2. It implies that probability mass shifts downwards as the sum of the values increases.

Example 1 (Inter-temporal Pricing with Privately Known Discount Rate). Consider the problem of inter-temporal pricing when the seller has commitment power: the seller owns an item that can be sold today or tomorrow. The buyer's value $v$ for receiving the item today, and $v \times \delta$, where $\delta \leq 1$ for receiving the item tomorrow. The seller can commit to a selling mechanism in advance, that could involve lotteries over the allocation of the item. Since the value for the item tomorrow is less than the value for the item today, a natural guess for the optimal mechanism is that it only allocates the item today. In other words, it posts a uniform price for both days, and the buyer will naturally select the item today (if at all). When $\delta$ is known to the seller, this intuition is proved correct in several related models such as Stokey (1979) and Acquisti and Varian (2005). For our model, this can be proved by simply applying Myerson's theorem, and noting that the virtual value for selling the item today multiplied by $\delta$ is equal to the virtual value for selling the item tomorrow.

When $\delta$ is only privately known to the buyer, however, uniform pricing is no longer generally optimal (Thanassoulis (2004) can be easily adopted to show this for our asymmetric problem). A direct application of our main theorem states that uniform pricing is optimal when $\delta$ and $v$ are affiliated, which includes but is more general than the case where $\delta$ and $v$ are independent. For instance, when $v$ is a uniform draw on the interval $[0,1]$ and is independent of $\delta$ the two dimensional the virtual value function that satisfies the amortization and single-dimensional-projection constraints is pinned down as $2 v-1$ for the item today and $\delta(2 v-1)$ for the item tomorrow. For $\delta \in[0,1]$ as defined, these virtual values satisfy are consistent with uniform pricing. Therefore, the existence of this virtual value function proves that uniform pricing is revenue optimal among all mechanisms.

For other i.i.d. distributions, the virtual values that amortization and single-dimensional-projection pin down do not generally satisfy consistency with uniform pricing (e.g., when values are i.i.d. $U[5,6]$ the optimal mechanism does not sell the agent his favorite item); however, we show that consistency 
with uniform pricing is satisfied by for multi-dimensional values that are i.i.d. from any distribution that satisfies a geometric convexity property.

As the second main example of the application of our framework, we provide sufficient conditions for optimality of grand bundle pricing for agents with additive preferences. The sum-ratio representation of a density function $f$ is the function $\bar{f}$ that is defined by

$$
f\left(t_{1}, t_{2}\right)=\bar{f}\left(t_{1}+t_{2}, t_{2} / t_{1}\right) .
$$

Theorem 2. Posting a price for the bundle of items is optimal if the sum-ratio representation of the density function is log-submodular, that is,

$$
\bar{f}(s, \theta) \times \bar{f}\left(s^{\prime}, \theta^{\prime}\right) \geq \bar{f}\left(s, \theta^{\prime}\right) \times \bar{f}\left(s^{\prime}, \theta\right), \quad s \leq s^{\prime}, \theta \geq \theta^{\prime} .
$$

Related Work. The starting point of work in multi-dimensional optimal mechanism design is the observation that an agent's utility must be a convex function of his private type (e.g., Rochet, 1985. cf. the envelope theorem). The second step is in writing revenue as the difference between the surplus of the mechanism and the agent's utility (e.g., McAfee and McMillan, 1988; Armstrong, 1996). The surplus can be expressed in terms of the gradient of the utility. The third step is in rewriting the objective in terms of either the utility (e.g., McAfee and McMillan, 1988; Manelli and Vincent, 2006; Hart and Nisan, 2013; Daskalakis et al., 2013; Wang and Tang, 2014; Giannakopoulos and Koutsoupias, 2014) or in terms of the gradient of the utility (e.g., Armstrong, 1996; Alaei et al., 2013 and this paper). This manipulation follows from an integration by parts. The first category of papers (rewriting objective in terms of utility) performs the integration by parts independently in each dimension, and the second category (rewriting objective in terms of gradient of utility, except for ours) does the integration along rays from the origin (see below). In our approach, in contrast, the integration by parts is performed in general and is dependent on the distribution and the form of the mechanism we wish to show is optimal.

Closest to our work are Armstrong (1996) and Alaei et al. (2013) which use integration by parts along paths that connect types with straight lines to the zero type (which has value zero for any outcome) to define virtual values. For the first work, Armstrong (1996) finds properties on valuation functions (beyond linear ones considered in our paper) and distributions, that when jointly satisfied, imply that the pointwise optimization of virtual values results in an incentive compatible mechanism. Armstrong gives some examples of mechanisms that result from this approach but does not generally interpret the form of the resulting mechanisms. Armstrong suggests generalizing his approach from rays from the origin to other kinds of paths; our approach, in contrast, proves the existence of appropriate paths over which to integrate without requiring the form of the path to be specified in advance. When Armstrong's condition on the distribution is satisfied (which we refer to as independence in max-ratio coordinates), our solution is also equivalent to an integration along rays. For the second work, Alaei et al. (2013) show that optimal multi-agent mechanism design can be reduced to optimal single-agent mechanism design by the construction of a single-dimensional virtual value (that satisfies similar properties to ours) when the single-agent mechanism design problems satisfy a revenue linearity property. They prove that a unit-demand agent with values for items that are independently, identically, and uniformly distributed on the $[0,1]$ interval is revenue linear; our results generalize this one. Moreover, our multi-dimensional virtual value construction constitutes a proof of revenue linearity; therefore, all of our optimal 
single-agent mechanisms automatically generalize to give optimal multi-agent mechanisms in the service constrained environments of Alaei et al. (2013).

There has been work looking at properties of single-agent mechanism design problems that are sufficient for optimal mechanisms to make only limited use of randomization. For context, the optimal single-item mechanism is always deterministic (e.g., Myerson, 1981; Riley and Zeckhauser, 1983), while the optimal multi-item mechanism is sometimes randomized (e.g., Thanassoulis, 2004 . Pycia, 2006). For agents with additive preferences across multiple items, McAfee and McMillan (1988), Manelli and Vincent (2006), and Giannakopoulos and Koutsoupias (2014) find sufficient conditions under which deterministic mechanisms, i.e., bundle pricings, are optimal. Pavlov (2011) considers more general preferences and a more general condition; for unit-demand preferences, this condition implies that in the optimal mechanism an agent deterministically receives an item or not, though the item received may be randomized.

A number of papers consider the question of finding closed forms for the optimal mechanism for an agent with additive preferences and independent values across the items. One such closed form is grand-bundle pricing. Our work for additive preferences contrasts in that we restrict to grandbundle pricing and a particular family of correlated distributions. For the two item case, Hart and Nisan (2013) give sufficient conditions for the optimality of grand-bundle pricing; these conditions are further generalized by Wang and Tang (2014). Their results are not directly comparable to ours as our results apply to correlated distributions. Daskalakis et al. (2013) and Giannakopoulos and Koutsoupias (2014) give frameworks, similar to ours, for proving optimality of multi-dimensional mechanisms. Daskalakis et al. (2013) use their framework to find the close form for the optimal mechanism for several special cases; Giannakopoulos and Koutsoupias (2014) give a closed form for the optimal mechanism when values are i.i.d. from the uniform distribution (with up to six items).

\section{Preliminaries}

\subsection{The Setting}

An agent is specified by a bounded set of possible types $T$ normalized to be $T=[0,1]^{m}$, where each $\boldsymbol{t}=\left(t_{1}, \ldots, t_{m}\right) \in T$ is an $m$-dimensional vector of values for $m$ items. ${ }^{4}$ The type of the agent is drawn from a known distribution with density $f$. For the special case that the type space is single-dimensional (i.e., $m=1$ ), the cumulative distribution function of the type is denoted by $F$. We do not require that the values for items be drawn independently. We consider unit-demand and additive agents. For unit-demand agents the allocation $\boldsymbol{x} \in[0,1]^{m}$ must satisfy $\sum_{i} x_{i} \leq 1$; for additive agents $\boldsymbol{x}$ must satisfy $x_{i} \leq 1$ for all $i$. The utility of the agent with type $\boldsymbol{t}$ for allocation $\boldsymbol{x} \in[0,1]^{m}$ and payment $p \in \mathbb{R}$ is $\boldsymbol{t} \cdot \boldsymbol{x}-p$.

A single-agent mechanism is a pair of functions, the allocation function $\boldsymbol{x}: T \rightarrow[0,1]^{m}$ and the payment function $p: T \rightarrow \mathbb{R}$. A mechanism is individually rational if the utility of every type of the agent is at least zero,

$$
\boldsymbol{t} \cdot \boldsymbol{x}(\boldsymbol{t})-p(\boldsymbol{t}) \geq 0, \quad \forall \boldsymbol{t}
$$

A mechanism is incentive compatible if no type of the agent increases his utility by misreporting,

\footnotetext{
${ }^{4}$ Throughout the paper we maintain the convention of denoting a vector $\mathbf{v}$ by a bold symbol and each of its components $v_{i}$ by a non-bold symbol.
} 


$$
\boldsymbol{t} \cdot \boldsymbol{x}(\boldsymbol{t})-p(\boldsymbol{t}) \geq \boldsymbol{t} \cdot \boldsymbol{x}(\hat{\boldsymbol{t}})-p(\hat{\boldsymbol{t}}), \quad \forall \boldsymbol{t}, \hat{\boldsymbol{t}}
$$

\subsection{Multivariable Calculus Notation}

For a function $h: \mathbb{R}^{k} \rightarrow \mathbb{R}$, we use $\partial_{j} h: \mathbb{R}^{k} \rightarrow \mathbb{R}$ to denote the partial derivative of function $h$ with respect to its $j$ 'th variable. The gradient of $h$ is a vector field, denoted by $\nabla h: \mathbb{R}^{k} \rightarrow \mathbb{R}^{k}$, defined to be $\nabla h=\left(\partial_{1} h, \ldots, \partial_{k} h\right)$. The divergence of a vector field $\boldsymbol{\alpha}: \mathbb{R}^{k} \rightarrow \mathbb{R}^{k}$ is denoted by $\nabla \cdot \boldsymbol{\alpha}: \mathbb{R}^{k} \rightarrow \mathbb{R}$ and is defined to be

$$
\nabla \cdot \boldsymbol{\alpha}=\partial_{1} \alpha_{1}+\ldots+\partial_{k} \alpha_{k}
$$

We denote the integral of function $h: \mathbb{R}^{k} \rightarrow \mathbb{R}$ over a subset $T$ of $\mathbb{R}^{k}$

$$
\int_{\boldsymbol{t} \in T} h(\boldsymbol{t}) \mathrm{d} \boldsymbol{t}
$$

Let $\partial T$ be the boundary of set $T$ and $\boldsymbol{\eta}(\boldsymbol{t})$ be the outward-pointing unit normal vector of $T$ at point $\boldsymbol{t}$ on $\partial T$. The multi-variable integration by parts for functions $h: \mathbb{R}^{k} \rightarrow \mathbb{R}$ and $\boldsymbol{\alpha}: \mathbb{R}^{k} \rightarrow \mathbb{R}^{k}$ is as follows

$$
\int_{\boldsymbol{t} \in T}(\nabla h \cdot \boldsymbol{\alpha})(\boldsymbol{t}) \mathrm{d} \boldsymbol{t}=\int_{\boldsymbol{t} \in \partial T} h(\boldsymbol{t})(\boldsymbol{\alpha} \cdot \boldsymbol{\eta})(\boldsymbol{t}) \mathrm{d} \boldsymbol{t}-\int_{\boldsymbol{t} \in T} h(\boldsymbol{t})(\nabla \cdot \boldsymbol{\alpha}(\boldsymbol{t})) \mathrm{d} \boldsymbol{t},
$$

Setting $h$ to be the constant function equal to 1 everywhere gives us the divergence theorem

$$
\int_{\boldsymbol{t} \in T}(\nabla \cdot \boldsymbol{\alpha})(\boldsymbol{t}) \mathrm{d} \boldsymbol{t}=\int_{\boldsymbol{t} \in \partial T}(\boldsymbol{\alpha} \cdot \boldsymbol{\eta})(\boldsymbol{t}) \mathrm{d} \boldsymbol{t} .
$$

When the dimension $k=1$, the integration by parts has the familiar form of

$$
\int_{x=a}^{b} h^{\prime}(x) g(x) \mathrm{d} x=\left.h(x) g(x)\right|_{x=a} ^{b}-\int_{x=a}^{b} h(x) g^{\prime}(x) \mathrm{d} x,
$$

and the divergence theorem is the fundamental theorem of calculus.

$$
\int_{x=a}^{b} h^{\prime}(x) \mathrm{d} x=h(b)-h(a) .
$$

\subsection{Problem Formulation}

A single agent mechanism $(\boldsymbol{x}, p)$ defines a utility function $u(\boldsymbol{t})=\boldsymbol{t} \cdot \boldsymbol{x}(\boldsymbol{t})-p(\boldsymbol{t})$. The following lemma connects the utility function of an IC mechanism with its allocation function.

Lemma 3 (Rochet, 1985). Function $u$ is the utility function of an agent in an incentive compatible mechanism if and only if $u$ is convex, and in that case, the agent's allocation is $\boldsymbol{x}(\boldsymbol{t})=\nabla u(\boldsymbol{t})$. 
Notice that the payment function can be defined using the utility function and the allocation function as $p(\boldsymbol{t})=\boldsymbol{t} \cdot \boldsymbol{x}(\boldsymbol{t})-u(\boldsymbol{t})$. Applying the above lemma, we can write payment to be $p(\boldsymbol{t})=\boldsymbol{t}$. $\nabla u(\boldsymbol{t})-u(\boldsymbol{t})$. The revenue maximization problem can then be written as the following mathematical program, which is the starting point for the analysis of this paper.

$$
\begin{aligned}
\max _{\boldsymbol{x}, u} & \int_{\boldsymbol{t}}[\boldsymbol{t} \cdot \nabla u(\boldsymbol{t})-u(\boldsymbol{t})] f(\boldsymbol{t}) \mathrm{d} \boldsymbol{t} \\
& \boldsymbol{x}=\nabla u ; u \text { is convex, } \\
& \forall \boldsymbol{t}, \boldsymbol{x}(\boldsymbol{t}) \text { is feasible allocation. }
\end{aligned}
$$

Notice that when the dimension of the type space is $m=1$, the above program is equivalent to the following familiar form from Myerson (1981),

$$
\begin{aligned}
\max _{x} & \int_{v}\left[v x(v)-\int_{z \leq v} x(z) \mathrm{d} z\right] f(v) \mathrm{d} v \\
& x \text { is monotone non-decreasing, } \\
& \forall v, x(v) \leq 1
\end{aligned}
$$

In Section 6, we extend the above formulation and our results to multi-agent settings.

\section{Amortization of Revenue}

This section formalizes our codification of multi-dimensional virtual values for incentive compatible mechanism design and describes the working pieces of our framework. The main construct is the definition of multi-dimensional virtual value functions and the accompanying proposition, below.

Definition 1. A vector field $\bar{\phi}:[0,1]^{m} \rightarrow \mathbb{R}^{m}$ that maps an an $m$-dimensional type to an $m$ dimensional vector is

- incentive compatible if the virtual surplus maximizer given by selecting the outcome $\boldsymbol{x}$ for type $\boldsymbol{t}$ that optimizes virtual surplus $\boldsymbol{x} \cdot \overline{\boldsymbol{\phi}}(\boldsymbol{t})$ is incentive compatible;

- a weak amortization of revenue if, in expectation over types drawn from the distribution, the virtual surplus of any incentive compatible mechanism upper bounds is revenue, i.e., $\mathbf{E}[\bar{\phi}(\boldsymbol{t}) \cdot \boldsymbol{x}(\boldsymbol{t})] \geq \mathbf{E}[p(\boldsymbol{t})]$, and with equality for the virtual surplus maximizer;

- a strong amortization of revenue if the inequality (of weak amortization) holds with equality for all incentive compatible mechanisms; and

- a virtual value function if it is incentive compatible and a weak or strong amortization.

Proposition 4. In any environment for which a virtual value function exists, the virtual surplus maximizer is incentive compatible and revenue optimal.

Proof. The expected revenue of the virtual surplus maximizer is equal to its expected virtual surplus (by weak amortization). This expected virtual surplus is at least the virtual surplus of any alternate mechanism (by definition of virtual surplus maximization). The expected virtual surplus is an upper bound on the expected revenue of the alternative mechanism (by weak amortization). Thus, the expected revenue of the virtual surplus maximizer is at least that of the alternative mechanism. Incentive compatibility follows directly from the definition of a virtual value function. 
For a single-dimensional agent (i.e., $m=1)$, Myerson (1981) showed that the function $v-\frac{1-F(v)}{f(v)}$ is a strong amortization, when it is monotone it is incentive compatible, when it is non-monotone an ironing procedure can be applied to obtain from it a weak amortization function that is monotone and thus incentive compatible. Our approach will analogously enable the derivation of multidimensional virtual value functions (i.e., satisfying incentive compatibility and weak amortization) via the construction of a strong amortization function that is not necessarily incentive compatible.

We now give sufficient conditions for a vector field to be a strong amortization of revenue. At a high level, we derive first a strong amortization of utility and then, using the fact that revenue is value minus utility, derive a strong amortization of revenue. These strong amortizations will be building blocks for the derivation of virtual values for unit-demand and additive agents in the subsequent sections. The following lemma follows from integration by parts as per equation (1), the definition of strong amortization of utility (Definition 1, generalized to utility), and the fact that the gradient of utility is the allocation rule of the mechanism (Lemma 3).

Lemma 5. For type space $T$ and distribution $f$, vector field $\boldsymbol{\alpha} / f$ is a strong amortization of utility, i.e., $\mathbf{E}[u(\boldsymbol{t})]=\mathbf{E}[\boldsymbol{\alpha}(\boldsymbol{t}) / f(\boldsymbol{t}) \cdot \boldsymbol{x}(\boldsymbol{t})]$ for all incentive compatible allocation rules $\boldsymbol{x}$, if it satisfies

- divergence density equality, i.e., that $\nabla \cdot \boldsymbol{\alpha}(\boldsymbol{t})=-f(\boldsymbol{t})$ at any point $\boldsymbol{t} \in T$, and

- boundary orthogonality, i.e., that $\boldsymbol{\alpha}(\boldsymbol{t}) \cdot \boldsymbol{\eta}(\boldsymbol{t})=0$ for all $\boldsymbol{t} \neq \mathbf{0}$ on the boundary of type space $\partial T$ with normal vector $\boldsymbol{\eta}(\boldsymbol{t})$.

Proof. Write the expectation of $\mathbf{E}[\boldsymbol{\alpha}(\boldsymbol{t}) / f(\boldsymbol{t}) \cdot \boldsymbol{x}(\boldsymbol{t})]$ as the integral $\int_{\boldsymbol{t} \in T} \boldsymbol{\alpha}(\boldsymbol{t}) \cdot \boldsymbol{x}(\boldsymbol{t}) \mathrm{d} \boldsymbol{t}$. From Lemma 3 , substitute $\nabla u$ for allocation $\boldsymbol{x}$ and apply integration by parts.

$$
\begin{aligned}
\int_{\boldsymbol{t} \in T} \boldsymbol{\alpha}(\boldsymbol{t}) \cdot \nabla u(\boldsymbol{t}) \mathrm{d} \boldsymbol{t} & =\int_{\boldsymbol{t} \in \partial T} u(\boldsymbol{t})(\boldsymbol{\alpha} \cdot \boldsymbol{\eta})(\boldsymbol{t}) \mathrm{d} \boldsymbol{t}-\int_{\boldsymbol{t} \in T} u(\boldsymbol{t})(\nabla \cdot \boldsymbol{\alpha}(\boldsymbol{t})) \mathrm{d} \boldsymbol{t} \\
& =u(\boldsymbol{t})+\int_{\boldsymbol{t} \in T} u(\boldsymbol{t}) f(\boldsymbol{t}) \mathrm{d} \boldsymbol{t}
\end{aligned}
$$

The second equality is derived from the first equality by employing the assumptions of the lemma on vector field $\boldsymbol{\alpha}$ as follows.

- By divergence density equality, the second term simplifies by substituting $\nabla \cdot \boldsymbol{\alpha}(\boldsymbol{t})=-f(\boldsymbol{t})$.

- Recall that the divergence theorem is equivalent setting $u(\boldsymbol{t})=1$ in the formula (3), this gives

$$
\int_{\boldsymbol{t} \in \partial T}(\boldsymbol{\alpha} \cdot \boldsymbol{\eta})(\boldsymbol{t}) \mathrm{d} \boldsymbol{t}=\int_{\boldsymbol{t} \in T} f(\boldsymbol{t}) \mathrm{d} \boldsymbol{t}=1,
$$

the total probability of any type. Boundary orthogonality implies that the integrand in the boundary integral of equation (5) is identically zero everywhere except $\boldsymbol{t}=0$. To integrate to one on the boundary, the function must be the Dirac delta function at $\boldsymbol{t}=0$; thus, the integral of the first term in equation $(3)$ is $u(\mathbf{0})$.

Without loss of generality for revenue optimal mechanisms $u(\mathbf{0})=0$. We can interpret the leftand right-hand sides of equation (4) as expectations, which gives $\mathbf{E}[\boldsymbol{\alpha}(\boldsymbol{t}) / f(\boldsymbol{t}) \cdot \boldsymbol{x}(\boldsymbol{t})]=\mathbf{E}[u(\boldsymbol{t})]$, the definition of strong amortization of utility for $\boldsymbol{\alpha} / f$. 
For a single-dimensional agent with value $v$ in type space $T=[0,1]$, the only function that satisfies the conditions of Lemma 5 and gives a strong amortization of utility is $\alpha(v) / f(v)=\frac{1-F(v)}{f(v)}$. For this formula, notice that the divergence of $\alpha(v)=1-F(v)$ is simply its derivative $-f(v)$. The boundary $\partial T \backslash\{0\}$ is the point $v=1$, the upper bound of the distribution, and thus trivially satisfies orthogonality as $\alpha(1)=0$. In classical auction theory the amortization of utility $\frac{1-F(v)}{f(v)}$ is often referred to as the agent's information rent.

The following lemma is immediate from the fact that revenue is the agent's surplus minus the agent's utility. For a single-dimensional agent it implies that $\phi(v)=v-\frac{(1-F(v))}{f(v)}$ is the strong amortization of revenue.

Lemma 6. For type space $T$ and distribution $f$, vector field $\boldsymbol{\phi}$ is a strong amortization of revenue, i.e., $\mathbf{E}[p(\boldsymbol{t})]=\mathbf{E}[\boldsymbol{\phi}(\boldsymbol{t}) \cdot \boldsymbol{x}(\boldsymbol{t})]$ for all incentive compatible allocation rules $\boldsymbol{x}$, if and only if $\boldsymbol{\phi}(\boldsymbol{t})=$ $\boldsymbol{t}-\boldsymbol{\alpha}(\boldsymbol{t}) / f(\boldsymbol{t})$ for all $\boldsymbol{t}$ but a measure zero subset of $T$ and $\boldsymbol{\alpha} / f$ is a strong amortization of utility.

Unlike the case of a single-dimensional agent, for multi-dimensional agents there are many strong amortizations of utility and, consequentally, many strong amortizations of revenue. As an example, suppose we wish to show the optimality of a restricted form of mechanism via a strongly amortized virtual value function for an $m=2$ dimensional agent. This virtual value fuction has two degrees of freedom. We can pin down one degree of freedom by equating virtual surplus to expected revenue for mechanisms with this restricted form. The divergence density equality for strong amortizations that Lemma 6 inherits from Lemma 5 gives a differential equation that then pins down the other degree of freedom. It remains to find sufficient conditions on the distribution under which virtual surplus maximization identically gives mechanisms of the restricted form.

The approach above can be generalized to show optimality of mechanisms via a weakly amortized vitual value function. For example, such a generalization can be used to give proofs of optimality under more permissive distributional assumptions. To subsitute weak amortization for stong amortization we need a way to relate the differential equations (from divergence density equality) that govern strong amortizations to any given weak amortization. Such a relationship follows directly from the definitions of both weak and strong amortization in terms of the expected revenue of any incentive compatible mechanism (Definition 1) and is summarized below as Lemma 7.

Lemma 7. For type space $T$ and distribution $f$, vector field $\bar{\phi}$ is a weak amortization of revenue if and only if there exists a strong amortization of revenue $\boldsymbol{\phi}$ such that $\mathbf{E}[\overline{\boldsymbol{\phi}}(\boldsymbol{t}) \boldsymbol{x}(\boldsymbol{t})] \geq \mathbf{E}[\boldsymbol{\phi}(\boldsymbol{t}) \boldsymbol{x}(\boldsymbol{t})]$, for all incentive compatible mechanisms $\boldsymbol{x}$, with equality for $\boldsymbol{x}$ that pointwise maximizes $\overline{\boldsymbol{\phi}}$.

\section{Optimality of Uniform Pricing for Unit Demand Preferences}

In this section we study the existence virtual values to prove optimality of uniform pricing for a single unit-demand agent. To simplify the exposition we focus on the case of two items and on the type space where item one is the favorite item, i.e., the lower right half of the unit square, $T=\left\{\boldsymbol{t}=\left(t_{1}, t_{2}\right): 0 \leq t_{2} \leq t_{1} \leq 1\right\}$. Our conclusions extend easily to the $[0,1]^{2}$ type space with symmetric disitributions; other extensions are given at the end of the section. The general case of $m \geq 2$ items is considered in Appendix A.

The single-dimensional projection for the favorite item is given by distribution and density function for the agent's favorite item, $F_{\max }(v)$ and $f_{\max }(v)$. The distribution function is the integral of $f$ over $\boldsymbol{t}$ with $t_{1} \geq v$. The density function is the integral of $f$ of $\boldsymbol{t}$ with $t_{1}=v$, i.e., $f_{\max }(v)=$ 
$\int_{0}^{v} f(v, z) \mathrm{d} z$. As described in Section 3, the unique strong amortization of revenue for a singledimensional agent (and thus for the single-dimensional projection) is $\phi_{\max }(v)=v-\frac{1-F_{\max }(v)}{f_{\max }(v)}$. The strong amortization of utility $\alpha_{\max } / f_{\max }$ requires $\alpha_{\max }(v)=1-F_{\max }(v)$.

Definition 2. The two-dimensional extension $\phi$ of the favorite-item projection $\phi_{\max }$ (satisfying $\left.\phi_{\max }(\boldsymbol{t})=t_{1}-\frac{1-F_{\max }\left(t_{1}\right)}{f_{\max }\left(t_{1}\right)}\right)$ is constructed as follows:

(a) Set $\phi_{1}(\boldsymbol{t})=\phi_{\max }\left(t_{1}\right)$ for all $\boldsymbol{t} \in T$.

(b) Let $\alpha_{1}(\boldsymbol{t})=\left(t_{1}-\phi_{1}(\boldsymbol{t})\right) f(\boldsymbol{t})=\frac{1-F_{\max }\left(t_{1}\right)}{f_{\max }\left(t_{1}\right)} f(\boldsymbol{t})$.

(c) Let $\alpha_{2}(\boldsymbol{t})=-\int_{y=0}^{t_{2}}\left(f\left(t_{1}, y\right)+\partial_{1} \alpha_{1}\left(t_{1}, y\right)\right) \mathrm{d} y$.

(d) Set $\phi_{2}(\boldsymbol{t})=t_{1}-\alpha_{2}(\boldsymbol{t}) / f(\boldsymbol{t})$.

In the remainder of this section we will show that for single-agent mechanism design to optimize revenue minus a fixed non-negative cost for selling either item, that this two-item extension of the favorite-item projection is a strong amortization of revenue that proves the optimality of uniform pricing: $5^{5}$

An informal justification of the steps of the construction is as follows:

(a) First, for fixed $t_{1}$ and as a function of $t_{2}, \phi_{1}(\boldsymbol{t})$ must be constant (i.e., on a vertical line in $T$ ); otherwise, there is a cost $c$ for which virtual surplus maximization with respect to $\phi$ serves one such type and not other is not the other which is not a uniform pricing. Second, the revenue of any mechanism that only ever sells the favorite item or nothing has revenue given by the favorite-item projection and must satisfy $\phi_{1}(\boldsymbol{t})=\phi_{\max }\left(t_{1}\right)$ (given the first point).

(b) We obtain $\alpha_{1}$ from $\phi_{1}$ by Lemma 6 . Orthogonality of the right boundary $\left(t_{1}=1\right)$ requires that $\boldsymbol{\alpha} \cdot(1,0)=0$, and therefore $\alpha_{1}\left(1, t_{2}\right)=0$. By definition, $\alpha_{1}\left(1, t_{2}\right)=\frac{\alpha_{\max }(1)}{f_{\max }(1)} f\left(1, t_{2}\right)=0$ and follows directly from boundary orthogonality of the favorite-item projection at $t_{1}=1$ which required $\alpha_{\max }(1)=0$ and was satisfied.

(c) The derivatives of $\alpha_{1}$ (with respect to $t_{1}$ ) and $\alpha_{2}$ (with respect to $t_{2}$ ) are related by the divergence density equality; integrating and employing boundary orthogonality on the bottom boundary $\left(t_{2}=0\right)$ of the type space, which requires that $\alpha_{2}\left(t_{2}, 0\right)=0$, gives the formula; these constraints are required by Lemma 5 .

(d) We obtain $\phi_{2}$ from $\alpha_{2}$ by Lemma 6 .

For $\phi$ to prove optimality of uniform pricing, it must be that virtual surplus maximization would never assign the agent the non-favorite item, i.e., item two. This requirement is simply $\phi_{1}(\boldsymbol{t}) \geq \phi_{2}(\boldsymbol{t})$ for any type $\boldsymbol{t} \in T$ for which either $\phi_{1}(\boldsymbol{t})$ or $\phi_{2}(\boldsymbol{t})$ is positive. A little algebra shows that this condition is implied by the angle of $\boldsymbol{\alpha}(\boldsymbol{t})$ being at most the angle of $\boldsymbol{t}$ (with respect to the horizontal $t_{2}$ axis; see Lemma 9. below). Importantly, in relation to the prior work of Armstrong (1996), the direction of $\boldsymbol{\alpha}$ corresponds to the paths on which incentive compatibility constraints are considered. The approach we are taking does not fix the direction, it allows any direction that

\footnotetext{
${ }^{5}$ The extra constraint imposed by a non-negative cost of service will enable this method to be extended to multiagent settings, see Section 6 This strong amortization is unique on the portion of type space for which $\phi_{1}(\boldsymbol{t})>0$.
} 


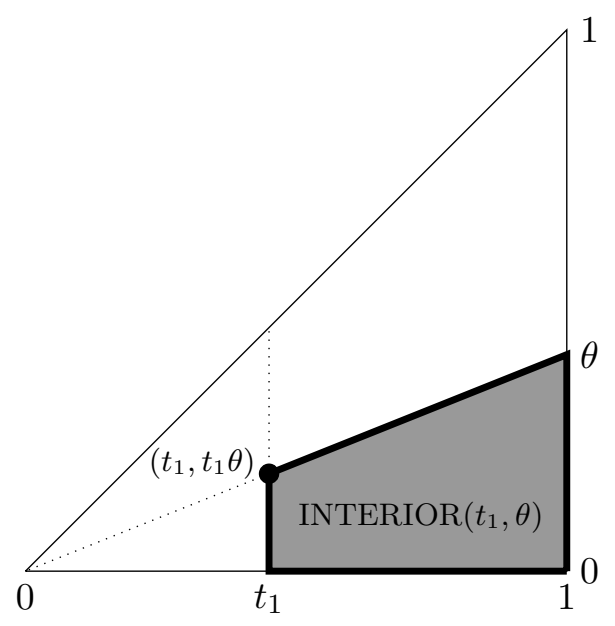

Figure 2: The trapezoidal set parameterized by $t_{1}$ and $\theta$, and the four curves that define its boundary, $\{$ TOP, RIGHT, BOTTOM, LEFT $\}\left(t_{1}, \theta\right)$

satisfies the above constraint on angles. The condition on angles is equvalant to the dot product between $\boldsymbol{\alpha}$ and the upward orthogonal vector to $\boldsymbol{t}$ being non-positive. The following lemma is proved by the divergence theorem.

Lemma 8. Vector field $\boldsymbol{\alpha} / f$ in the definition of the two-dimensional extension of the favorite-item projection is a strong amortization of utility and satisfies

$$
\theta \alpha_{1}\left(t_{1}, t_{1} \theta\right)-\alpha_{2}\left(t_{1}, t_{1} \theta\right)=\left(1-F_{\max }\left(t_{1}\right)\right) \frac{\mathrm{d}}{\mathrm{d} t_{1}}\left[\frac{\int_{t_{2}^{\prime}=0}^{t_{1} \theta} f\left(t_{1}, t_{2}^{\prime}\right) \mathrm{d} t_{2}^{\prime}}{f_{\max }\left(t_{1}\right)}\right]
$$

for all $t_{1}, \theta \in[0,1]$ (and thus $\left(t_{1}, t_{1} \theta\right) \in T$ ).

Proof. The informal justifications of Steps (b) and (c) show that $\boldsymbol{\alpha}$ satisfies the divergence density equality and bottom and right boundary orthogonality. This proof starts with these assumption and derives the identity of the lemma. Notice that for $\theta=1$ the numerator and the denomenator in the derivative of the identity are equal for all $t_{1}$, the right-hand side is zero, and therefore $\alpha_{1}\left(t_{1}, t_{1}\right)=\alpha_{2}\left(t_{1}, t_{1}\right)$, and boundary orthogonality holds for the diagonal boundary. Thus, $\boldsymbol{\alpha} / f$ is a strong amortization of utility.

The strategy for the proof of the identity is as follows. We fix $t_{1}$ and $\theta$ and apply the divergence theorem to $\boldsymbol{\alpha}$ on the trapezoidal subspace of type space defined by types $\boldsymbol{t}^{\prime}$ with $t_{1}^{\prime} \geq t_{1}, t_{2}^{\prime} / t_{1}^{\prime} \leq \theta$, $t_{2}^{\prime} \geq 0$, and $t_{1}^{\prime} \leq 1$ (Figure 2). The divergence theorem equates the the integral of the vector field $\boldsymbol{\alpha}$ on the boundary of the subspace to the integral of its divergence within the subspace. As the upper boundary of this trapesoidal subspace has slope $t_{2} / t_{1}$, one term in this equality is the integral of $\boldsymbol{\alpha}\left(\boldsymbol{t}^{\prime}\right)$ with the upward orthogonal vector to $\boldsymbol{t}$. Differentiating this integral and evaluating at $\boldsymbol{t}^{\prime}=\left(t_{1}, t_{1} \theta\right)$ gives the desired quantity.

Appling the divergence theorem to $\boldsymbol{\alpha}$ on the trapezoid and experessing the top boundary as the interior divergence minus the other three boundaries gives:

$\int_{\boldsymbol{t}^{\prime} \in \mathrm{TOP}\left(t_{1}, \theta\right)} \boldsymbol{\eta}\left(\boldsymbol{t}^{\prime}\right) \cdot \boldsymbol{\alpha}\left(\boldsymbol{t}^{\prime}\right) \mathrm{d} \boldsymbol{t}^{\prime}=\int_{\boldsymbol{t}^{\prime} \in \operatorname{INTERIOR}\left(t_{1}, \theta\right)} \nabla \cdot \boldsymbol{\alpha}\left(\boldsymbol{t}^{\prime}\right) \mathrm{d} \boldsymbol{t}^{\prime}-\int_{\boldsymbol{t}^{\prime} \in\{\mathrm{RIGHT}, \text { BOTTOM }, \mathrm{LEFT}\}\left(t_{1}, \theta\right)} \boldsymbol{\eta}\left(\boldsymbol{t}^{\prime}\right) \cdot \boldsymbol{\alpha}\left(\boldsymbol{t}^{\prime}\right) \mathrm{d} \boldsymbol{t}^{\prime}$. 
Since $\boldsymbol{\alpha} / f$ is a strong amortization of utility, the divergence density equality and boundary orthogonality of Lemma 5 imply that the integral over the interior simplifies and the integrals over the right and bottom boundary are zero, respectively. We have,

$\int_{\boldsymbol{t}^{\prime} \in \mathrm{TOP}\left(t_{1}, \theta\right)} \boldsymbol{\eta}\left(\boldsymbol{t}^{\prime}\right) \cdot \boldsymbol{\alpha}\left(\boldsymbol{t}^{\prime}\right) \mathrm{d} \boldsymbol{t}^{\prime}=-\int_{\boldsymbol{t}^{\prime} \in \operatorname{INTERIOR}\left(t_{1}, \theta\right)} f\left(\boldsymbol{t}^{\prime}\right) \mathrm{d} \boldsymbol{t}^{\prime}-\int_{\boldsymbol{t}^{\prime} \in \operatorname{LEFT}\left(t_{1}, \theta\right)} \boldsymbol{\eta}\left(\boldsymbol{t}^{\prime}\right) \cdot \boldsymbol{\alpha}\left(\boldsymbol{t}^{\prime}\right) \mathrm{d} \boldsymbol{t}^{\prime}$.

For the trapezoid at $\boldsymbol{t}$ these integrals are,

$$
\begin{aligned}
\int_{t_{1}^{\prime}=t_{1}}^{1}\left(-\theta \alpha_{1}\left(t_{1}^{\prime}, t_{1}^{\prime} \theta\right)\right. & \left.+\alpha_{2}\left(t_{1}^{\prime}, t_{1}^{\prime} \theta\right)\right) \mathrm{d} t_{1}^{\prime} \\
& =-\int_{t_{1}^{\prime}=t_{1}}^{1} \int_{t_{2}^{\prime}=0}^{t_{1}^{\prime} \theta} f\left(\boldsymbol{t}^{\prime}\right) \mathrm{d} t_{2}^{\prime} \mathrm{d} t_{1}^{\prime}+\int_{t_{2}^{\prime}=0}^{t_{1} \theta} \alpha_{1}\left(t_{1}, t_{2}^{\prime}\right) \mathrm{d} t_{2}^{\prime} .
\end{aligned}
$$

Differentiating with respect to $t_{1}$ gives,

$$
\theta \alpha_{1}\left(t_{1}, t_{1} \theta\right)-\alpha_{2}\left(t_{1}, t_{1} \theta\right)=\int_{t_{2}^{\prime}=0}^{t_{1} \theta} f\left(t_{1}, t_{2}^{\prime}\right) \mathrm{d} t_{2}^{\prime}+\frac{\mathrm{d}}{\mathrm{d} t_{1}} \int_{t_{2}^{\prime}=0}^{t_{1} \theta} \alpha_{1}\left(t_{1}, t_{2}^{\prime}\right) \mathrm{d} t_{2}^{\prime}
$$

On the right-hand side, multiply first term by $\frac{f_{\max }\left(t_{1}\right)}{f_{\max }\left(t_{1}\right)}=1$ and plug in the strong amortization of utility for the two-dimensional extension as $\alpha(\boldsymbol{t})=\frac{1-F_{\max }\left(t_{1}\right)}{f_{\max }\left(t_{1}\right)} f(\boldsymbol{t})$ to the second term. Notice that the integral of the second term is only on $t_{2}^{\prime}$ therefore we can bring the terms related to $t_{1}$ outside the integral. These two terms then simplify by the product rule for differentiation to give the identity of the lemma.

$$
\begin{aligned}
\theta \alpha_{1}\left(t_{1}, t_{1} \theta\right)-\alpha_{2}\left(t_{1}, t_{1} \theta\right) & =f_{\max }\left(t_{1}\right) \frac{\int_{t_{2}^{\prime}=0}^{t_{1} \theta} f\left(t_{1}, t_{2}^{\prime}\right) \mathrm{d} t_{2}^{\prime}}{f_{\max }\left(t_{1}\right)}+\frac{\mathrm{d}}{\mathrm{d} t_{1}}\left[\left(1-F_{\max }\left(t_{1}\right)\right) \frac{\int_{t_{2}^{\prime}=0}^{t_{1} \theta} f\left(t_{1}, t_{2}^{\prime}\right) \mathrm{d} t_{2}^{\prime}}{f_{\max }\left(t_{1}\right)}\right] . \\
& =\left(1-F_{\max }\left(t_{1}\right)\right) \frac{\mathrm{d}}{\mathrm{d} t_{1}}\left[\frac{\int_{t_{2}^{\prime}=0}^{t_{1} \theta} f\left(t_{1}, t_{2}^{\prime}\right) \mathrm{d} t_{2}^{\prime}}{f_{\max }\left(t_{1}\right)}\right] .
\end{aligned}
$$

Lemma 9. For $\boldsymbol{\phi}$ and $\boldsymbol{\alpha}$ defined by the two-dimensional extension of the favorite-item projection, if $\frac{t_{2}}{t_{1}} \alpha_{1}\left(t_{1}, t_{2}\right)-\alpha_{2}\left(t_{1}, t_{2}\right)$ is non-positive and $\phi_{1}(\boldsymbol{t})$ is monotone non-decreasing in $\boldsymbol{t}$, then virtual surplus maximization with respect to $\phi$ and any non-negative service cost c gives a uniform pricing.

Proof. From the assumption $\frac{t_{2}}{t_{1}} \alpha_{1}\left(t_{1}, t_{2}\right)-\alpha_{2}\left(t_{1}, t_{2}\right) \leq 0$ and Definition 2 we have

$$
\frac{t_{2}}{t_{1}} \phi_{1}(\boldsymbol{t})=\frac{t_{2}}{t_{1}}\left(t_{1}-\frac{\alpha_{1}(\boldsymbol{t})}{f(\boldsymbol{t})}\right)=\frac{t_{2}}{t_{1}}\left(t_{1}-\frac{\alpha_{1}(\boldsymbol{t})}{f(\boldsymbol{t})}\right) \geq t_{2}-\frac{\alpha_{2}(\boldsymbol{t})}{f(\boldsymbol{t})}=\phi_{2}(\boldsymbol{t}) .
$$

Thus, for $\boldsymbol{t}$ with $\phi_{1}(\boldsymbol{t}) \geq c, \phi_{1}(\boldsymbol{t}) \geq \phi_{2}(\boldsymbol{t})$ and virtual surplus maximization serves the agent item one. Since $\phi_{1}(\boldsymbol{t})$ is a function only of $t_{1}$ (Definition 2), its monotonicity implies that there is a smallest $t_{1}$ such that all greater types are served. Also, if $\phi_{1}(\boldsymbol{t}) \leq c$, again the above calculation implies that $\phi_{2}(\boldsymbol{t}) \leq c$ and therefore the type is not served. This outcome is one of a uniform pricing. 
We are now ready to state the main theorems of this section. In the next section we will give an interpretation of the main technical condition as a supermodularity condition on the density function.

Theorem 10. Uniform pricing is revenue optimal for any service cost $c$ and any distribution for which the favorite-item projection has monotone non-decreasing strong amortization $\bar{\phi}_{\max }\left(t_{1}\right)=$ $t_{1}-\frac{1-F_{\max }\left(t_{1}\right)}{f_{\max }\left(t_{1}\right)}$ and $\frac{\mathrm{d}}{\mathrm{d} t_{1}}\left[\frac{1}{f_{\max }\left(t_{1}\right)} \int_{0}^{t_{2}} f\left(t_{1}, y\right) \mathrm{d} y\right]$ is non-positive.

The conditions of Corollary 13 can be further relaxed by constructing a weak amortization $\bar{\phi}$ from the strong amortization $\phi$, above. The following is a special case of the more general Lemma 24 given in Section 6.3.

Theorem 11. Uniform pricing is revenue optimal for any service cost $c$ and any distribution for which $\frac{\mathrm{d}}{\mathrm{d} t_{1}}\left[\frac{1}{f_{\max }\left(t_{1}\right)} \int_{0}^{t_{2}} f\left(t_{1}, y\right) \mathrm{d} y\right]$ is non-positive.

In Appendix A we employ using the natural generalization of the condition to extend the above results to $m \geq 2$ items.

\subsection{Max-ratio log-supermodular distributions}

Theorem 11 and Theorem 10 in the preceding section require the non-positivity of $\frac{\mathrm{d}}{\mathrm{d} t_{1}}\left[\frac{1}{f_{\max }\left(t_{1}\right)} \int_{0}^{t_{2}} f\left(t_{1}, y\right) \mathrm{d} y\right]$ which is not easy to directly check; in this section we give a simpler sufficient condition. To specify the condition, it is useful to view the distribution in the max-ratio (MR) coordinates. In particular the max-ratio representation $f^{M R}$ of a density function $f$ is defined to be

$$
f^{M R}\left(t_{1}, \theta\right)=f\left(t_{1}, \theta t_{1}\right), \quad \forall t_{1}, \theta, 0 \leq t_{1}, \theta \leq 1 .
$$

Equivalantly,

$$
f\left(t_{1}, t_{2}\right)=f^{M R}\left(t_{1}, t_{2} / t_{1}\right) \quad \forall t_{1}, t_{2}, 0 \leq t_{1}, t_{2} \leq 1 .
$$

We call a distribution MR-log-supermodular, if its max-ratio representation is log-supermodular,

$$
f^{M R}\left(t_{1}, \theta\right) \times f^{M R}\left(t_{1}^{\prime}, \theta^{\prime}\right) \geq f^{M R}\left(t_{1}, \theta^{\prime}\right) \times f^{M R}\left(t_{1}^{\prime}, \theta\right), \quad \forall t_{1} \leq t_{1}^{\prime}, \theta \leq \theta^{\prime} .
$$

Notice that, for example, MR-independent distributions are MR-log-supermodular. A MRindependent distribution $f^{M R}$ is such that $f^{M R}\left(t_{1}, \theta_{1}\right)=f_{1}\left(t_{1}\right) \times f_{\theta}(\theta)$ (for arbitrary $f_{1}$ and $f_{\theta}$ ), and is MR-log-supermodular because

$$
\begin{aligned}
f^{M R}\left(t_{1}, \theta\right) \times f^{M R}\left(t_{1}^{\prime}, \theta^{\prime}\right) & =f_{1}\left(t_{1}\right) f_{\theta}(\theta) f_{1}\left(t_{1}^{\prime}\right) f_{\theta}\left(\theta^{\prime}\right) \\
& =f_{1}\left(t_{1}\right) f_{\theta}\left(\theta^{\prime}\right) f_{1}\left(t_{1}^{\prime}\right) f_{\theta}(\theta) \\
& =f^{M R}\left(t_{1}, \theta^{\prime}\right) \times f^{M R}\left(t_{1}^{\prime}, \theta\right) .
\end{aligned}
$$

We now prove that $\frac{\mathrm{d}}{\mathrm{d} t_{1}}\left[\frac{1}{f_{\max }\left(t_{1}\right)} \int_{0}^{t_{2}} f\left(t_{1}, y\right) \mathrm{d} y\right]$ is non-positive for MR-log-supermodular distributions. This result enables the interpretation of Theorem 10 in terms of MR-log-supermodularity. Recall that the density function of the favorite-item projection is defined from the two-item density function as $f_{\max }\left(t_{1}\right)=\int_{0}^{t_{1}} f\left(t_{1}, y\right) \mathrm{d} y$. 
Lemma 12. If $f$ is a MR-log-supermodular function, then for any $t_{1}$ and $\theta$,

$$
\frac{\mathrm{d}}{\mathrm{d} t_{1}}\left[\frac{\int_{t_{2}^{\prime}=0}^{t_{1} \theta} f\left(t_{1}, t_{2}^{\prime}\right) \mathrm{d} t_{2}^{\prime}}{\int_{t_{2}^{\prime}=0}^{t_{1}} f\left(t_{1}, t_{2}^{\prime}\right) \mathrm{d} t_{2}^{\prime}}\right] \leq 0,
$$

with equality if distribution is MR-independent.

Proof. We prove that for any $\theta, t_{1}$, and $t_{1}^{\prime}$ such that $t_{1}<t_{1}^{\prime}$,

$$
\frac{\int_{t_{2}^{\prime}=0}^{t_{1} \theta} f\left(t_{1}, t_{2}^{\prime}\right) \mathrm{d} t_{2}^{\prime}}{\int_{t_{2}^{\prime}=0}^{t_{1}} f\left(t_{1}, t_{2}^{\prime}\right) \mathrm{d} t_{2}^{\prime}} \leq \frac{\int_{t_{2}^{\prime}=0}^{t_{1}^{\prime} \theta} f\left(t_{1}^{\prime}, t_{2}^{\prime}\right) \mathrm{d} t_{2}^{\prime}}{\int_{t_{2}^{\prime}=0}^{t_{1}^{\prime}} f\left(t_{1}^{\prime}, t_{2}^{\prime}\right) \mathrm{d} t_{2}^{\prime}} .
$$

The proof first converts the above form into max-ratio coordinates, applies MR-log-supermodularity, and then transforms back to the standard form. Before applying MR-log-supermodularity, we break down the integral set into two set, and apply MR-log-supermodularity to only one of the integrals. More particularly, notice that

$$
\begin{aligned}
& \int_{t_{2}^{\prime}=0}^{t_{1} \theta} f\left(t_{1}, t_{2}^{\prime}\right) \mathrm{d} t_{2}^{\prime} \times \int_{t_{2}^{\prime}=0}^{t_{1}^{\prime}} f\left(t_{1}^{\prime}, t_{2}^{\prime}\right) \mathrm{d} t_{2}^{\prime} \\
& =\int_{\theta^{\prime}=0}^{\theta} f^{M R}\left(t_{1}, \theta^{\prime}\right) t_{1} \mathrm{~d} \theta^{\prime} \times \int_{\theta^{\prime \prime}=0}^{1} f^{M R}\left(t_{1}^{\prime}, \theta^{\prime \prime}\right) t_{1}^{\prime} \mathrm{d} \theta^{\prime \prime} \quad \text { (change of variables) } \\
& =\int_{\theta^{\prime}=0}^{\theta} \int_{\theta^{\prime \prime}=0}^{\theta} f^{M R}\left(t_{1}, \theta^{\prime}\right) t_{1} f^{M R}\left(t_{1}^{\prime}, \theta^{\prime \prime}\right) t_{1}^{\prime} \mathrm{d} \theta^{\prime \prime} \mathrm{d} \theta^{\prime} \\
& +\int_{\theta^{\prime}=0}^{\theta} \int_{\theta^{\prime \prime}=\theta}^{1} f^{M R}\left(t_{1}, \theta^{\prime}\right) t_{1} f^{M R}\left(t_{1}^{\prime}, \theta^{\prime \prime}\right) t_{1}^{\prime} \mathrm{d} \theta^{\prime \prime} \mathrm{d} \theta^{\prime} \quad \text { (separate double integral into two sets) } \\
& \left.\leq \int_{\theta^{\prime}=0}^{\theta} \int_{\theta^{\prime \prime}=0}^{\theta} f^{M R}\left(t_{1}, \theta^{\prime \prime}\right) t_{1} f^{M R}\left(t_{1}^{\prime}, \theta^{\prime}\right) t_{1}^{\prime} \mathrm{d} \theta^{\prime} \mathrm{d} \theta^{\prime \prime} \quad \text { (rename variables } \theta^{\prime} \text { and } \theta^{\prime \prime}\right) \\
& +\int_{\theta^{\prime}=0}^{\theta} \int_{\theta^{\prime \prime}=\theta}^{1} f^{M R}\left(t_{1}, \theta^{\prime \prime}\right) t_{1} f^{M R}\left(t_{1}^{\prime}, \theta^{\prime}\right) t_{1}^{\prime} \mathrm{d} \theta^{\prime \prime} \mathrm{d} \theta^{\prime} \quad \text { (apply MR-log-supermodularity) } \\
& =\int_{\theta^{\prime \prime}=0}^{1} f^{M R}\left(t_{1}, \theta^{\prime \prime}\right) t_{1} \mathrm{~d} \theta^{\prime \prime} \int_{\theta^{\prime}=0}^{\theta} f^{M R}\left(t_{1}^{\prime}, \theta^{\prime}\right) t_{1}^{\prime} \mathrm{d} \theta^{\prime} \quad \text { (merge integrals) } \\
& =\int_{t_{2}^{\prime}=0}^{t_{1}} f\left(t_{1}, t_{2}^{\prime}\right) \mathrm{d} t_{2}^{\prime} \times \int_{t_{2}^{\prime}=0}^{t_{1}^{\prime} \theta} f\left(t_{1}^{\prime}, t_{2}^{\prime}\right) \mathrm{d} t_{2}^{\prime} .
\end{aligned}
$$

By combining the above lemma and Corollary 13 we get the following corollary.

Corollary 13. Uniform pricing is revenue optimal for any service cost $c$ and any max-ratio logsupermodular distribution.

To understand MR-log-supermodular distributions better, we next show properties under which product distributions are MR-log-supermodular. We call a function $g$ geometric-geometric (GG) convex if

$$
g\left(z_{1}^{\lambda} z_{2}^{1-\lambda}\right) \geq g\left(z_{1}\right)^{\lambda} g\left(z_{2}\right)^{1-\lambda}, \quad \forall \lambda \in[0,1], z_{1}, z_{2}
$$


For example, the function $g(x)=x^{k}$ is GG-convex. More generally, for any convex function $h$ and constant $c$, the function $g(x)=c \cdot e^{h(\log (x))}$ is GG-convex. The proof of the following lemma can be found in Appendix B.

Lemma 14. The product distribution on i.i.d. draws from a distirbution with geometric-geometric convex density is max-ratio log-supermodular.

\subsection{Bundle Pricing for General Distributions}

We can use the generality of unit-demand settings and the above result to find conditions under which bundle pricing is optimal for general distributions. More precisely, notice that any problem with finite outcome space can be converted to a unit-demand problem with dot-product utility function by letting $\boldsymbol{t}$ and $\boldsymbol{x}$ be vectors of size equal to the size of the outcome space, with $t_{i}$ being the value for outcome $i$. For multi-item settings with $m$ items, the size of the outcome space is equal to $2^{m}$. The input to the problem is a density function $f\left(t_{1}, \ldots, t_{k}\right)$ over vectors of size $k=2^{m}$. We assume that the density function is monotone, meaning that the density of a type is non-zero only if the valuation for the grand bundle is at least as much as the value for any other subset of items. The max-ratio representation of the density function is $\bar{f}\left(t_{1}, \theta_{2}, \ldots, \theta_{k}\right)$, where $t_{1}$, normalized to be at most 1 , is the value for the grand bundle, and $\theta_{i} \leq 1$ is the ratio of the value for the $i$-th subset, over the value of the grand bundle. Now a bundle pricing mechanism corresponds to a mechanism in the unit demand setting which only sells the favorite outcome, which is the grand bundle. We therefore have the following theorem.

Theorem 15. Bundle pricing is optimal if the max-ratio representation of the density function is MR-log-supermodular.

\section{Optimality of Bundle Pricing for Additive Preferences}

In this section we provide sufficient conditions for optimality of grand bundle pricing for agents with additive utilities. Similar to Section 4, we only focus on constructing a proof assuming that item one is the favorite item, as the proof generalizes to symmetric distributions easily by mirroring the construction for the other half of set of types. It is also easiest to express the results of this section when the sum of the values for the items are normalized to be at most one. We thus define the set of types to be $T=\left\{\left(t_{1}, t_{2}\right) \mid t_{1}, t_{2} \geq 0, t_{1} \geq t_{2}, t_{1}+t_{2} \leq 1\right\}$.

The single-dimensional projection for the sum of values is given by distribution and density function for the agent's favorite item, $F_{\text {sum }}(v)$ and $f_{\text {sum }}(v)$. The distribution function is the integral of $f$ over $\boldsymbol{t}$ with $t_{1}+t_{2} \leq v$. The density function $f_{\text {sum }}(v)$ is the derivative of $F_{\text {sum }}(v)$ with respect to $v$. As described in Section 3 , the unique strong amortization of revenue for a single-dimensional agent (and thus for the single-dimensional projection) is $\phi_{\text {sum }}(v)=v-\frac{1-F_{\text {sum }}(v)}{f_{\text {sum }}(v)}$. The strong amortization of utility $\alpha_{\text {sum }} / f_{\text {sum }}$ requires $\alpha_{\text {sum }}(v)=1-F_{\text {sum }}(v)$.

In this section, we prove optimality of grand bundle pricing by constructing a weak amortization of revenue $\bar{\phi}$ (as opposed to Section 4 in which we directly constructed a strong amortization), which we call the two-dimensional extension of the sum-of-values projection. We will first define $\bar{\phi}$ such that the virtual surplus of bundle pricing with respect to $\bar{\phi}$ is equal to its revenue (Lemma 16), for which grand bundle pricing maximizes virtual surplus (Lemma 17). The rest of the section proves that $\bar{\phi}$ is indeed a weak amortization of revenue by using Lemma 7 to show existence of a 
strong amortization which lower bounds the virtual surplus, with respects to $\bar{\phi}$, of any incentive compatible mechanism.

Define $\bar{\phi}_{1}$ and $\bar{\phi}_{2}$ as follows

$$
\begin{aligned}
& \bar{\phi}_{1}(\boldsymbol{t})=\frac{t_{1}}{t_{1}+t_{2}} \phi_{\text {sum }}\left(t_{1}+t_{2}\right)=t_{1}-\frac{1-F_{\text {sum }}\left(t_{1}+t_{2}\right)}{f_{\text {sum }}\left(t_{1}+t_{2}\right)}, \\
& \bar{\phi}_{2}(\boldsymbol{t})=\frac{t_{2}}{t_{1}+t_{2}} \phi_{\text {sum }}\left(t_{1}+t_{2}\right)=t_{2}-\frac{1-F_{\text {sum }}\left(t_{1}+t_{2}\right)}{f_{\text {sum }}\left(t_{1}+t_{2}\right)} .
\end{aligned}
$$

This definition ensures that $\bar{\phi}$ satisfies two important properties. First, the virtual surplus of bundle pricing is equal to the virtual surplus of the single-dimensional projection for sum of values, that is, $\bar{\phi}_{1}(\boldsymbol{t})+\bar{\phi}_{2}(\boldsymbol{t})=\phi_{\text {sum }}\left(t_{1}+t_{2}\right)$. As a result of this property, the virtual surplus of grand bundle pricing can be shown to be equal to its revenue (see the next lemma). Second, the two components of the virtual value function have the same sign, which together with the monotonicity of $\phi_{\text {sum }}$ would imply that bundle pricing maximizes virtual surplus (Lemma 17).

Lemma 16 (single-dimensional projection). The expected revenue of a bundle pricing is equal to its expected virtual surplus with respect to the two-dimensional extension of the sum-of-values projection $\overline{\boldsymbol{\phi}}$ satisfying $\bar{\phi}_{1}(\boldsymbol{t})+\bar{\phi}_{2}(\boldsymbol{t})=\phi_{\text {sum }}\left(t_{1}+t_{2}\right)$ where $\phi_{\text {sum }}(v)=v-\frac{1-\bar{F}_{\text {sum }}(v)}{f_{\text {sum }}(v)}$ is the strong amortization for agent's sum-of-values projection.

Functions $\bar{\phi}_{1}$ and $\bar{\phi}_{2}$ have the same sign as $\phi_{\text {sum }}$, and therefore as long as $\phi_{\text {sum }}$ is a nondecreasing function of $p$, there exists a price $p^{*}$ such that the allocation corresponding to price $p^{*}$ ex-post optimizes $\bar{\phi}$, according to the following lemma.

Lemma 17 (consistency with virtual surplus maximization; incentive compatibility). Virtual surplus maximization according to vector field $\bar{\phi}$ gives a bundle pricing $p$ (and is incentive compatible) if and only if

- $\bar{\phi}_{1}(\boldsymbol{t}), \bar{\phi}_{2}(\boldsymbol{t}) \geq 0$ when $t_{1}+t_{2} \geq p$ and $\bar{\phi}_{1}(\boldsymbol{t}), \bar{\phi}_{2}(\boldsymbol{t}) \leq 0$ otherwise.

Proof. We need to show that for the uniform price $p$, the allocation function $\boldsymbol{x}$ of posting a price $p$ for the bundle optimizes $\boldsymbol{\phi}$ pointwise. Pointwise optimization of $\boldsymbol{x} \cdot \overline{\boldsymbol{\phi}}$ will result in $\boldsymbol{x}=(1,1)$ whenever $\bar{\phi}_{1}, \bar{\phi}_{2} \geq 0$, and will result in $\boldsymbol{x}=(0,0)$ whenever $\phi_{1}, \phi_{2} \leq 0$.

In order to complete the proof we should ensure that $\bar{\phi}$ is an amortization of revenue. We already constructed $\bar{\phi}$ such that the expected virtual surplus of the virtual surplus maximizer, bundle pricing, is equal to its revenue. So we only need to show that the virtual surplus of any incentive compatible allocation is at least its revenue. We do this by constructing a strong amortization of revenue $\phi$ and invoking Lemma 7. That is, we will show that there exists a strong amortization $\phi$ such that for any incentive compatible allocation $\boldsymbol{x}$,

$$
\int_{\boldsymbol{t}} \boldsymbol{x}(\boldsymbol{t}) \cdot[\bar{\phi}(\boldsymbol{t})-\boldsymbol{\phi}(\boldsymbol{t})] f(\boldsymbol{t}) \mathrm{d} \boldsymbol{t} \geq 0
$$

To do this, it would be sufficient to ensure that $\bar{\phi}_{1} \geq \phi_{1}$ and $\bar{\phi}_{2} \geq \phi_{2}$ for any type. Instead, in Lemma 18 we suggest a more permissive sufficient condition using integration by parts, and the properties of incentive compatible allocations. Lemma 20 later defines conditions that would imply 
that a strong amortization satisfying the conditions of Lemma 18 can be constructed, using an approach very similar to the proof of Lemma 8.

For a function $h$ on type space $T$, define $h^{S R}$ to be its transformation to sum-ratio coordinates, that is

$$
h\left(t_{1}, t_{2}\right)=h^{S R}\left(t_{1}+t_{2}, \frac{t_{2}}{t_{1}}\right)
$$

Sum-ratio coordinates are convenient to work with since it allows us to refer to a type using its value for the bundle, and the ratio of values of item one and item two. The proof of the following lemma first transforms the expression in inequality (6) to sum-ratio coordinates, and invokes singledimensional integration by parts along the lines with constant sum of values to get an expression in terms of derivative of $\boldsymbol{x}$ times a (weighted) integral of $\overline{\boldsymbol{\phi}}-\boldsymbol{\phi}$ (see expression (7)). We then use a property of incentive compatible allocations to verify the sign of the given expression.

Lemma 18. The two-dimensional extension of the sum-of-values projection $\bar{\phi}$ is a weak amortization of revenue if there exists a strong amortization $\phi$ such that

1. $\phi_{1}(\boldsymbol{t})+\phi_{2}(\boldsymbol{t})=\phi_{\text {sum }}\left(t_{1}+t_{2}\right)$.

2. $\phi_{1}(\boldsymbol{t}) \frac{t_{2}}{t_{1}} \leq \phi_{2}(\boldsymbol{t})$.

Proof. We invoke Lemma 7 and construct a strong amortization $\phi$ that shows that the expected virtual surplus, with respect to $\bar{\phi}$ of any incentive compatible allocation is at least its revenue. Thus we need to show that the following expression for all incentive compatible $\boldsymbol{x}$,

$$
\int_{\boldsymbol{t}} \boldsymbol{x}(\boldsymbol{t}) \cdot[\bar{\phi}(\boldsymbol{t})-\boldsymbol{\phi}(\boldsymbol{t})] f(\boldsymbol{t}) \mathrm{d} \boldsymbol{t} \geq 0 .
$$

By symmetry of the distribution, the optimal mechanism is also symmetric. Therefore, we prove the lemma only for symmetric incentive compatible allocations (in particular, we assume that $x_{1}\left(t_{1}, t_{1}\right)=x_{2}\left(t_{1}, t_{1}\right)$ for all $\left.t_{1}\right)$. Perform a change of variables $s=t_{1}+t_{2}$, and $\theta=t_{2} / t_{1}$ on the left hand side of the above expression (6)

$$
\begin{aligned}
& \int_{s=0}^{1} \int_{\theta=0}^{1} \boldsymbol{x}^{S R}(s, \theta) \cdot[\overline{\boldsymbol{\phi}}-\boldsymbol{\phi}]^{S R}(s, \theta) f^{S R}(s, \theta) \frac{s}{1+\theta} \mathrm{d} \theta \mathrm{d} s \\
& =\int_{s=0}^{1} \int_{\theta=0}^{1} \boldsymbol{x}^{S R}(s, \theta) \cdot \frac{d}{d \theta} \int_{\theta^{\prime}=0}^{\theta}[\overline{\boldsymbol{\phi}}-\boldsymbol{\phi}]^{S R}\left(s, \theta^{\prime}\right) f^{S R}\left(s, \theta^{\prime}\right) \frac{s}{1+\theta^{\prime}} \mathrm{d} \theta^{\prime} \mathrm{d} \theta \mathrm{d} s .
\end{aligned}
$$

To simplify exposition, let

$$
\gamma(s, \theta)=\int_{\theta^{\prime}=0}^{\theta}[\overline{\boldsymbol{\phi}}-\boldsymbol{\phi}]^{S R}\left(s, \theta^{\prime}\right) f^{S R}\left(s, \theta^{\prime}\right) \mathrm{d} \theta^{\prime},
$$

to rewrite the left hand side of inequality (6)

$$
\begin{aligned}
& =\int_{s=0}^{1} \int_{\theta=0}^{1} \boldsymbol{x}^{S R}(s, \theta) \cdot \frac{d}{d \theta} \gamma(s, \theta) \mathrm{d} \theta \mathrm{d} s \\
& =\left.\int_{s=0}^{1} \boldsymbol{x}^{S R}(s, \theta) \cdot \gamma(s, \theta)\right|_{\theta=0} ^{1}-\int_{\theta=0}^{1} \frac{d}{d \theta} \boldsymbol{x}^{S R}(s, \theta) \cdot \gamma(s, \theta) \mathrm{d} \theta \mathrm{d} s
\end{aligned}
$$


using integration by parts. The first term of the above expression is zero. For $\theta=0$, we have $\underline{\gamma}=0$ since the interval of integration is empty. For $\theta=1, x_{1}(s, 1)=x_{2}(s, 1)$ by symmetry, and $\bar{\phi}_{1}-\phi_{1}+\bar{\phi}_{2}-\phi_{2}=0$ by the assumption of the lemma. Therefore, the left hand side of inequality (6) simplifies to

$$
=-\int_{s=0}^{1} \int_{\theta=0}^{1} \frac{d}{d \theta} \boldsymbol{x}^{S R}(s, \theta) \cdot \gamma(s, \theta) \mathrm{d} \theta \mathrm{d} s .
$$

Lemma 19 shows that $-\frac{d}{d \theta} \boldsymbol{x}^{S R}(s, \theta) \cdot(1,-1) \geq 0$, and therefore we can complete the proof by showing that $\gamma_{1}=-\gamma_{2}$ and $\gamma_{1} \geq 0$ everywhere. The first property holds by the first assumption of the lemma, since $\phi_{1}+\phi_{2}=\phi_{\text {sum }}\left(t_{1}+t_{2}\right)=\bar{\phi}_{1}+\bar{\phi}_{2}$ implies that $\bar{\phi}_{1}-\phi_{1}=\phi_{2}-\bar{\phi}_{2}$. Also, $\phi_{1}+\phi_{2}=\bar{\phi}_{1}+\bar{\phi}_{2}$ together with $\bar{\phi}_{1} \frac{t_{2}}{t_{1}}=\bar{\phi}_{2}$ (by definition) and $\phi_{1} \frac{t_{2}}{t_{1}} \leq \phi_{2}$ (by assumption of the lemma) implies that $\bar{\phi}_{1} \geq \phi_{1}$.

Lemma 19. The allocation of any incentive compatible mechanism satisfies

$$
\frac{d}{d \theta} \boldsymbol{x}^{S R}(s, \theta) \cdot(-1,1) \geq 0
$$

Proof. Incentive compatibility implies that for any $s, \theta$, and $\epsilon$,

$$
\left(\boldsymbol{x}^{S R}(s, \theta+\epsilon)-\boldsymbol{x}^{S R}(s, \theta)\right) \cdot(-1,1) \frac{\theta \epsilon}{s} \geq 0 .
$$

Letting $\epsilon$ approach zero implies the claim.

So in order to complete the proof that $\bar{\phi}$ is a weak amortization, we should construct strong amortization $\phi$ satisfying conditions of Lemma 18. Recall that $\alpha_{1} \frac{t_{2}}{t_{1}} \geq \alpha_{2}$ implies that $\phi_{1} \frac{t_{2}}{t_{1}} \leq \phi_{2}$ because

$$
\frac{t_{2}}{t_{1}} \phi_{1}(\boldsymbol{t})=\frac{t_{2}}{t_{1}}\left(t_{1}-\frac{\alpha_{1}(\boldsymbol{t})}{f(\boldsymbol{t})}\right)=\frac{t_{2}}{t_{1}}\left(t_{1}-\frac{\alpha_{1}(\boldsymbol{t})}{f(\boldsymbol{t})}\right) \leq t_{2}-\frac{\alpha_{2}(\boldsymbol{t})}{f(\boldsymbol{t})}=\phi_{2}(\boldsymbol{t}) .
$$

So the goal is to construct $\phi$ such that $\alpha_{1} \frac{t_{2}}{t_{1}} \geq \alpha_{2}$. Extend the definition of the projection for the sum of values as follows. Define $F_{\text {sum }}(v, \theta)$ to be the probability of the set of types $\boldsymbol{t}$ such that $t_{1}+t_{2} \geq v$, and $t_{2} / t_{1} \leq \theta$. This implies that $F_{\text {sum }}(v)=F_{\text {sum }}(v, 1)$. Define $f_{\text {sum }}(v, \theta)$ to be the density function of the distribution, that is $f_{\text {sum }}(v, \theta)=\frac{\mathrm{d}}{\mathrm{d} v} F_{\text {sum }}(v, \theta)$.

Lemma 20. There exists unique strong amortization $\boldsymbol{\phi}=t f-\boldsymbol{\alpha}$ satisfying $\phi_{1}+\phi_{2}=\phi_{\text {sum }}\left(t_{1}+t_{2}\right)$. This amortization also satisfies

$$
\theta \alpha_{1}^{S R}(s, \theta)-\alpha_{2}^{S R}(s, \theta)=-(1+\theta)\left(1-F_{\text {sum }}(s)\right) \frac{\mathrm{d}}{\mathrm{d} s}\left[\frac{f_{\text {sum }}(s, \theta)}{f_{\text {sum }}(s)}\right] .
$$

Proof. We will here sketch the proof of the above lemma, and the complete proof appears in Appendix B.

The proof is similar to the proof of Lemma 8, and takes the derivative of the divergence theorem. We assume $\phi$ exists, use the divergence theorem and properties of $\phi$ to derive a closed form for it, 


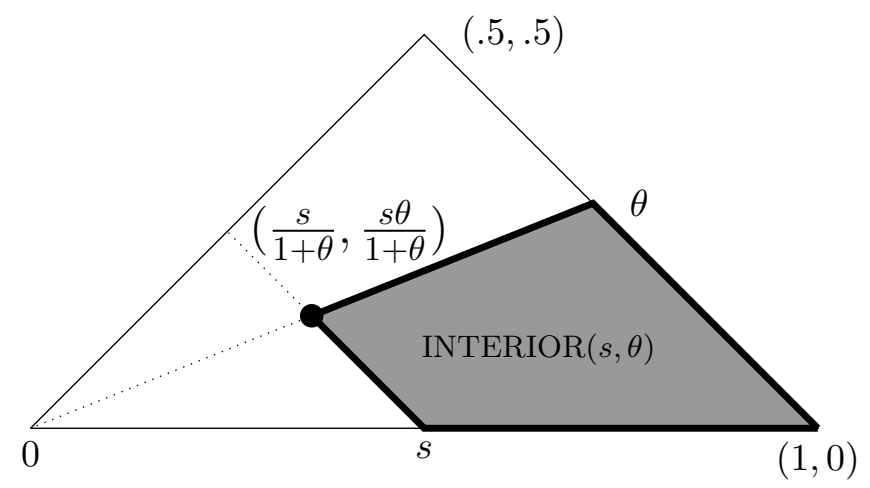

Figure 3: The trapezoidal set parameterized by $s$ and $\theta$, and the four curves that define its boundary, $\{$ TOP, RIGHT, BOTTOM, LEFT $\}(s, \theta)$

and then verify that $\phi$ indeed satisfies all the required properties. We apply the divergence theorem to $\boldsymbol{\alpha}$ on the trapezoidal subspace of type space defined by types $\boldsymbol{t}^{\prime}$ with $t_{1}^{\prime}+t_{2}^{\prime} \geq s, t_{2}^{\prime} / t_{1}^{\prime} \leq \theta$, $t_{2}^{\prime} \geq 0$, and $t_{1}^{\prime}+t_{2}^{\prime} \leq 1$ (Figure 3). The divergence theorem equates the integral of the vector field $\boldsymbol{\alpha}$ on the boundary of the subspace to the integral of its divergence within the subspace. The integral of the vector field over right and bottom boundaries equates zero by boundary orthogonality, and its value over the left boundary will be specified given the equation $\phi_{1}+\phi_{2}=\phi_{\text {sum }}\left(t_{1}+t_{2}\right)$ (the outward pointing vector is $(-1,-1))$. As the upper boundary of this trapezoidal subspace has slope $t_{2} / t_{1}$, one term in this equality is the integral of $\boldsymbol{\alpha}\left(\boldsymbol{t}^{\prime}\right)$ with the upward orthogonal vector to $\boldsymbol{t}$. Differentiating this integral and evaluating at $\boldsymbol{t}^{\prime}=\left(\frac{s}{1+\theta}, \frac{s \theta}{1+\theta}\right)$ gives a closed form expression for $\frac{t_{2}}{t_{1}} \alpha_{1}-\alpha_{2}$. We then verify that the specified $\phi$ satisfies all the properties.

A distribution $f$ is SR-log-submodular if it is log-submodular in sum-ratio coordinates, that is,

$$
f^{S R}(s, \theta) \times f^{S R}\left(s^{\prime}, \theta^{\prime}\right) \geq f^{S R}\left(s^{\prime}, \theta\right) \times f^{S R}\left(s, \theta^{\prime}\right), \quad \forall s \leq s^{\prime}, \theta \leq \theta^{\prime},
$$

and is SR-independent if the above holds with equality everywhere. Similar to Section 4 we prove the following lemma (notice that the sign is the opposite of the sign in Section 4, which is the reason that supermodularity is replaced by sub modularity).

Lemma 21. If $f$ is a $S R$-log-submodular function, then for any $s$ and $\theta$,

$$
\frac{\mathrm{d}}{\mathrm{d} s}\left[\frac{f_{\text {sum }}(s, \theta)}{f_{\text {sum }}(s)}\right] \geq 0,
$$

with equality if distribution is SR-independent.

Theorem 22. Bundle pricing is revenue optimal for any SR-log-submodular distribution for which the sum-of-values projection has monotone strong amortization $\phi_{\text {sum }}$.

\section{Multi Agent Extensions}

\subsection{The Setting}

A multi-agent problem is defined by $n$ agents, each agent $\kappa$ associated with a distribution $f^{\kappa}$, and a feasibility setting $\mathcal{S} \subseteq\{0,1\}^{n \times m}$. Types of agents are drawn independent of each other 
from corresponding distributions. A multi-agent mechanism is a sequence of allocation functions $\left(\overline{\boldsymbol{x}}^{1}\left(\boldsymbol{t}^{1}, \ldots, \boldsymbol{t}^{n}\right), \ldots, \overline{\boldsymbol{x}}^{n}\left(\boldsymbol{t}^{1}, \ldots, \boldsymbol{t}^{m}\right)\right) \in \mathcal{S}$ in which $\overline{\boldsymbol{x}}^{\kappa}\left(\boldsymbol{t}^{1}, \ldots, \boldsymbol{t}^{n}\right) \in \mathbb{R}^{m}$ is the allocation of agent $\kappa$, together with a sequence of payment function $\left(p^{1}\left(\boldsymbol{t}^{1}, \ldots, \boldsymbol{t}^{n}\right), \ldots, p^{n}\left(\boldsymbol{t}^{1}, \ldots, \boldsymbol{t}^{n}\right)\right) \in \mathbb{R}^{n}$ in which $p^{\kappa}\left(\boldsymbol{t}^{1}, \ldots, \boldsymbol{t}^{n}\right) \in \mathbb{R}$ is the payment of agent $\kappa$. A multi-agent mechanism is ex-post individually rational (EPIR) if the utility of every type of every agent is always positive,

$$
\boldsymbol{t}^{\kappa} \cdot \overline{\boldsymbol{x}}^{\kappa}\left(\boldsymbol{t}^{\kappa}, \boldsymbol{t}^{-\kappa}\right)-p^{\kappa}\left(\boldsymbol{t}^{\kappa}, \boldsymbol{t}^{-\kappa}\right) \geq 0, \quad \forall \kappa, \boldsymbol{t}^{\kappa}, \boldsymbol{t}^{-\kappa}
$$

where $\boldsymbol{t}^{-\kappa}$ is the vector of other agents types. A multi-agent mechanism is dominant strategy incentive compatible (DSIC) if no type of any agent increases his utility by misreporting,

$$
\boldsymbol{t}^{\kappa} \cdot \overline{\boldsymbol{x}}^{\kappa}\left(\boldsymbol{t}^{\kappa}, \boldsymbol{t}^{-\kappa}\right)-p^{\kappa}\left(\boldsymbol{t}^{\kappa}, \boldsymbol{t}^{-\kappa}\right) \geq \boldsymbol{t}^{\kappa} \cdot \overline{\boldsymbol{x}}^{\kappa}\left(\hat{\boldsymbol{t}}^{\kappa}, \boldsymbol{t}^{-\kappa}\right)-p^{\kappa}\left(\hat{\boldsymbol{t}}^{\kappa}, \boldsymbol{t}^{-\kappa}\right), \quad \forall \kappa, \boldsymbol{t}^{\kappa}, \hat{\boldsymbol{t}}^{\kappa}, \boldsymbol{t}^{-\kappa}
$$

The interim mechanism for agent $\kappa$ is a single-agent mechanism $\left(\boldsymbol{x}^{\kappa}, p^{\kappa}\right)$ defined to be

$$
\begin{aligned}
\boldsymbol{x}^{\kappa}\left(\boldsymbol{t}^{\kappa}\right) & =E_{\boldsymbol{t}^{-\kappa} \sim f^{-\kappa}}\left[\overline{\boldsymbol{x}}^{\kappa}\left(\boldsymbol{t}^{\kappa}, \boldsymbol{t}^{-\kappa}\right)\right], \\
p^{\kappa}\left(\mathbf{t}^{\kappa}\right) & =E_{\boldsymbol{t}^{-\kappa} \sim f^{-\kappa}}\left[p^{\kappa}\left(\boldsymbol{t}^{\kappa}, \boldsymbol{t}^{-\kappa}\right)\right],
\end{aligned}
$$

where $\boldsymbol{t}^{-\kappa}$ is the vector of other agents types, drawn at random from $f^{-\kappa}:=f^{1} \times \ldots \times f^{\kappa-1} \times$ $f^{\kappa+1} \times \ldots \times f^{n}$. A mechanism is interim individually rational (IIR) if the interim mechanism of every agent is individually rational. A mechanism is Bayesian incentive compatible (BIC) if the interim mechanism of every agent is incentive compatible. Notice that EPIR implies IIR, and DSIC implies BIC. The goal is to design feasible IIC and IIR mechanisms that maximize expected payments. We however prove stronger results that the optimal mechanisms are in fact EPIR and DSIC.

\subsection{Problem Formulation}

A single agent mechanism $(\boldsymbol{x}, p)$ defines a utility function $u(\boldsymbol{t})=\boldsymbol{t} \cdot \boldsymbol{x}(\boldsymbol{t})-p(\boldsymbol{t})$. Recall the following lemma from Section 2.3 which connects the utility function of an IC mechanism with its allocation function.

Lemma 3 (Rochet (1985)). Function $u$ is the utility function of an agent in an incentive compatible mechanism if and only if $u$ is convex, and in that case, the agent's allocation is $\boldsymbol{x}(\boldsymbol{t})=\nabla u(\boldsymbol{t})$.

The payment function can be defined using the utility function and the allocation function as $p(\boldsymbol{t})=\boldsymbol{t} \cdot \boldsymbol{x}(\boldsymbol{t})-u(\boldsymbol{t})$. Using the above lemma, we can write payment to be $p(\boldsymbol{t})=\boldsymbol{t} \cdot \nabla u(\boldsymbol{t})-u(\boldsymbol{t})$. This allows us to write the following optimization problem as our revenue maximization problem, which is the starting point of the analysis of this paper.

$$
\begin{aligned}
\max _{\overline{\boldsymbol{x}}^{1}, \ldots, \overline{\boldsymbol{x}}^{n}, u^{1}, \ldots, u^{n}} & \sum_{\kappa} \int_{\boldsymbol{t}}\left[\boldsymbol{t} \cdot \nabla u^{\kappa}(\boldsymbol{t})-u^{\kappa}(\boldsymbol{t})\right] f^{\kappa}(\boldsymbol{t}) \mathrm{d} \boldsymbol{t} \\
& \forall \kappa, \boldsymbol{x}^{\kappa}=\nabla u^{\kappa} ; u^{\kappa} \text { is convex, } \\
& \forall \boldsymbol{t},\left(\overline{\boldsymbol{x}}^{1}\left(\boldsymbol{t}^{1}, \ldots, \boldsymbol{t}^{n}\right), \ldots, \overline{\boldsymbol{x}}^{n}\left(\boldsymbol{t}^{1}, \ldots, \boldsymbol{t}^{n}\right)\right) \text { is feasible allocation. }
\end{aligned}
$$


Notice that when $m=1$, the above program is equivalent to the following familiar form of

$$
\begin{aligned}
\max _{\overline{\boldsymbol{x}}^{1}, \ldots, \overline{\boldsymbol{x}}^{n}} & \sum_{i} \int_{v}\left[v \boldsymbol{x}^{\kappa}(v)-\int_{z \leq v} \boldsymbol{x}^{\kappa}(z) d z\right] f(v) \mathrm{d} v \\
& \forall \kappa, \boldsymbol{x}^{\kappa} \text { is monotone non-decreasing, } \\
& \forall \boldsymbol{t},\left(\overline{\boldsymbol{x}}^{1}\left(\boldsymbol{t}^{1}, \ldots, \boldsymbol{t}^{n}\right), \ldots, \overline{\boldsymbol{x}}^{n}\left(\boldsymbol{t}^{1}, \ldots, \boldsymbol{t}^{n}\right)\right) \text { is feasible allocation. }
\end{aligned}
$$

Now assume that for each agent $\kappa$, we have constructed $\boldsymbol{\alpha}^{\kappa}(\boldsymbol{t})$ according to Lemma 5 . The above analysis and Program 8 tells us that we can write revenue as

$$
\begin{aligned}
\sum_{\kappa} \int_{\boldsymbol{t}^{\kappa}} \boldsymbol{x}^{\kappa}\left(\boldsymbol{t}^{\kappa}\right) \cdot \boldsymbol{\phi}^{\kappa}\left(\boldsymbol{t}^{\kappa}\right) f^{\kappa}\left(\boldsymbol{t}^{\kappa}\right) \mathrm{d} \boldsymbol{t}^{\kappa} & =\sum_{\kappa} \int_{\boldsymbol{t}^{\kappa}}\left[\int_{\boldsymbol{t}^{-\kappa}} \overline{\boldsymbol{x}}^{\kappa}\left(\boldsymbol{t}^{\kappa}, \boldsymbol{t}^{-\kappa}\right) f^{-\kappa}\left(\boldsymbol{t}^{-\kappa}\right) \mathrm{d} \boldsymbol{t}^{-\kappa}\right] \cdot \boldsymbol{\phi}^{\kappa}\left(\boldsymbol{t}^{\kappa}\right) f^{\kappa}\left(\boldsymbol{t}^{\kappa}\right) \mathrm{d} \boldsymbol{t}^{\kappa} \\
& =\sum_{\kappa} \int_{\boldsymbol{t}} \overline{\boldsymbol{x}}^{\kappa}\left(\boldsymbol{t}^{\kappa}, \boldsymbol{t}^{-\kappa}\right) \cdot \boldsymbol{\phi}^{\kappa}\left(\boldsymbol{t}^{\kappa}\right) f(\boldsymbol{t}) \mathrm{d} \boldsymbol{t} \\
& =\int_{\boldsymbol{t}}\left[\sum_{\kappa} \overline{\boldsymbol{x}}^{\kappa}\left(\boldsymbol{t}^{\kappa}, \boldsymbol{t}^{-\kappa}\right) \cdot \boldsymbol{\phi}^{\kappa}\left(\boldsymbol{t}^{\kappa}\right)\right] f(\boldsymbol{t}) \mathrm{d} \boldsymbol{t} .
\end{aligned}
$$

We say that a mechanism $\overline{\boldsymbol{x}}$ optimizes an objective $\boldsymbol{\phi}$ ex-post, if for any input $\boldsymbol{t}^{1}, \ldots, \boldsymbol{t}^{m}$ it selects an outcome that maximizes $\sum_{\kappa} \overline{\boldsymbol{x}}^{\kappa}\left(\boldsymbol{t}^{1}, \ldots, \boldsymbol{t}^{m}\right) \cdot \boldsymbol{\phi}^{\kappa}\left(\boldsymbol{t}^{\kappa}\right)$.

Notice that in a multi-agent problem, the existence of $\boldsymbol{\alpha}^{\kappa}$ depends only on the distribution $f^{\kappa}$. As a result, in the rest of this section we study construction of $\boldsymbol{\alpha}^{\kappa}$ for single agents, and drop the index $\kappa$. By showing the existence of proofs for each agent separately, we can apply the meta theorem to get the structure of the multi-agent optimal solution.

\subsection{Ex-post Optimization and Ironing}

Having proved that $\phi$ is a strong amortization of revenue by proving it satisfies conditions of Lemma 5, we now study its incentive compatibility in multi-agent settings. First we define serviceconstrained settings. A service-constrained environment is characterized by a set system $\mathcal{S} \subseteq 2^{n}$. An allocation is feasible if $\left\{i \mid \sum_{j=m} x_{i j}=1\right\} \in \mathcal{S}$. A service-constrained environment is downwardclosed if for any $S, S^{\prime} \subseteq S$ such that $S \in \mathcal{S}$, we also have $S^{\prime} \in \mathcal{S}$.

Notice that $\phi_{2} \leq \frac{t_{2}}{t_{1}} \phi_{1}$ implies that when $\phi_{1} \geq 0$, we have $\phi_{2} \leq \phi_{1}$, and when $\phi_{1} \leq 0$, we also have $\phi_{2} \leq 0$. This implies that in service-constrained settings, an ex-post optimizer of $\phi$ will assign positive probability only to the favorite item. In addition, if $\phi(t)$ is a non-decreasing function of $t_{1}$, then so will be the allocation probability for the favorite item. This implies that the allocation rule is incentive compatible because

$$
\begin{aligned}
\left(\boldsymbol{x}(\boldsymbol{t})-\boldsymbol{x}\left(\boldsymbol{t}^{\prime}\right)\right) \cdot\left(\boldsymbol{t}-\boldsymbol{t}^{\prime}\right) & =\left(\boldsymbol{x}_{1}(\boldsymbol{t})-\boldsymbol{x}_{1}\left(\boldsymbol{t}^{\prime}\right)\right)\left(t_{1}-t_{1}^{\prime}\right) \\
& \geq 0,
\end{aligned}
$$

which shows the convexity of the utility function, which in turn proves incentive compatibility (see Lemma 3). We have therefore proved the following lemma. 
Lemma 23. If $\boldsymbol{\phi}(\boldsymbol{t})$ is a non-decreasing function of $t_{1}$, and $\phi_{1} \geq \phi_{2}$ whenever $\phi_{2} \geq 0$, then $\boldsymbol{\phi}$ is incentive compatible for any multi-agent downward-closed service-constrained environment.

Unfortunately, as it happens in single-dimensional settings, the derivative of revenue is not necessarily monotone. However, we can fix this problem by ironing the virtual value function in a similar manner to the single-dimensional problem. An ironing of multi-dimensional virtual values should be performed carefully in order to keep the properties of virtual values. More precisely, we would like to maintain the property that $\phi_{2} \leq \phi_{1}$ when $\phi_{2} \geq 0$. In particular, we can prove the following lemma (proof is in Appendix B).

Lemma 24. There exists an ironed virtual value function $\bar{\phi}$ that is a weak amortization of revenue and is incentive compatible (by satisfying the conditions of Lemma 23) for MR-log-supermodular distributions.

The above lemma directly implies our main theorem.

Theorem 25. In multi-agent downward-closed service-constrained environments with MR-log-supermodular distributions, the optimal auction selects a feasible set maximizing the sum of virtual values for favorite items.

\section{References}

Acquisti, A. and Varian, H. R. (2005). Conditioning prices on purchase history. Marketing Science, $24(3): 367-381$.

Alaei, S., Fu, H., Haghpanah, N., and Hartline, J. (2013). The simple economics of approximately optimal auctions. In FOCS.

Armstrong, M. (1996). Multiproduct nonlinear pricing. Econometrica, 64(1):51-75.

Armstrong, M. (1999). Price discrimination by a many-product firm. The Review of Economic Studies, 66(1):151-168.

Daskalakis, C., Deckelbaum, A., and Tzamos, C. (2013). Mechanism design via optimal transport. In Proceedings of the fourteenth ACM conference on Electronic commerce, pages 269-286. ACM.

Giannakopoulos, Y. and Koutsoupias, E. (2014). Duality and optimality of auctions for uniform distributions. In ACM Conference on Electronic Commerce.

Hart, S. and Nisan, N. (2013). The menu-size complexity of auctions. arXiv preprint arXiv:1304.6116.

Manelli, A. M. and Vincent, D. R. (2006). Bundling as an optimal selling mechanism for a multiplegood monopolist. Journal of Economic Theory, 127(1):1-35.

McAfee, R. P. and McMillan, J. (1988). Multidimensional incentive compatibility and mechanism design. Journal of Economic Theory, 46(2):335-354.

Myerson, R. (1981). Optimal auction design. Mathematics of Operations Research, 6(1):pp. 58-73. 
Pavlov, G. (2011). A property of solutions to linear monopoly problems. The BE Journal of Theoretical Economics, 11(1).

Pycia, M. (2006). Stochastic vs deterministic mechanisms in multidimensional screening.

Riley, J. and Zeckhauser, R. (1983). Optimal selling strategies: When to haggle, when to hold firm. The Quarterly Journal of Economics, 98(2):267-289.

Rochet, J. (1985). The taxation principle and multi-time hamilton-jacobi equations. Journal of Mathematical Economics, 14(2):113 - 128.

Stokey, N. L. (1979). Intertemporal price discrimination. The Quarterly Journal of Economics, pages 355-371.

Thanassoulis, J. (2004). Haggling over substitutes. J. Economic Theory, 117(2):217-245.

Wang, Z. and Tang, P. (2014). Optimal mechanisms with simple menus. In ACM Conference on Electronic Commerce.

\section{A Extension for $m>2$ Items}

In this section, we extend the definition of $\boldsymbol{\alpha}$ given in Section 4 from two items to $m>2$. The approach to derive such general $\boldsymbol{\alpha}$ is similar to that of Section 4 , and therefore in this section we only define $\boldsymbol{\alpha}$ and verify its properties. Assume that we are in a partition of the space in which $t_{1} \geq t_{i}$ for all $i$. Define $T\left(t_{1}, \theta_{2}, \ldots, \theta_{m}\right)=\left\{\boldsymbol{t}^{\prime} \mid t_{1}^{\prime} \geq t_{1}, t_{i}^{\prime} \leq \theta_{i} t_{1}, \forall i \geq 2\right\}$. Define $C\left(t_{1}, \theta_{2}, \ldots, \theta_{m}\right)=$ $\left\{\boldsymbol{t}^{\prime} \mid t_{1}^{\prime}=t_{1}, t_{i}^{\prime} \leq t_{i}^{\prime} \theta_{i}, \forall i \geq 2\right\}$. Now define

$$
\alpha_{1}(\boldsymbol{t})=t_{1}-\frac{\int_{\boldsymbol{t}^{\prime} \in T\left(t_{1}, 1, \ldots, 1\right)} f\left(\boldsymbol{t}^{\prime}\right) \mathrm{d} \boldsymbol{t}^{\prime}}{\int_{\boldsymbol{t}^{\prime} \in C\left(t_{1}, 1, \ldots, 1\right)} f\left(\boldsymbol{t}^{\prime}\right) \mathrm{d} \boldsymbol{t}^{\prime}}
$$

We also define $\alpha_{i}$ for $i \geq 2$ as follows

$$
\alpha_{i}(\boldsymbol{t}) f(\boldsymbol{t})=t_{i} f(\boldsymbol{t})+\frac{1}{m-1} \int_{y=0}^{t_{i}} f\left(y, \boldsymbol{t}_{-i}\right)+\frac{d}{d t_{1}}\left[\left(t_{1}-\alpha_{1}\left(y, \boldsymbol{t}_{-i}\right)\right) f\left(y, \boldsymbol{t}_{-i}\right)\right] \mathrm{d} y
$$

The above definition implies that

$$
\frac{d}{d t_{i}}\left(t_{i} f(\boldsymbol{t})-\alpha_{i}(\boldsymbol{t}) f(\boldsymbol{t})\right)=-\frac{1}{m-1}\left(f(\boldsymbol{t})+\frac{d}{d t_{1}}\left[\left(t_{1}-\alpha_{1}(\boldsymbol{t})\right) f(\boldsymbol{t})\right]\right) .
$$

As a result,

$$
\begin{aligned}
\nabla \cdot \phi & =\sum_{i} \frac{d}{d t_{i}}\left(t_{i} f(\boldsymbol{t})-\alpha_{i}(\boldsymbol{t}) f(\boldsymbol{t})\right) \\
& =\frac{d}{d t_{1}}\left[\left(t_{1}-\alpha_{1}(\boldsymbol{t})\right) f(\boldsymbol{t})\right]-(m-1) \times \frac{1}{m-1}\left(f(\boldsymbol{t})+\frac{d}{d t_{1}}\left[\left(t_{1}-\alpha_{1}(\boldsymbol{t})\right) f(\boldsymbol{t})\right]\right) \\
& =-f
\end{aligned}
$$


We now verify that $\phi$ satisfies the boundary conditions. This holds because when $t_{i}=0$, $\alpha_{i}(\boldsymbol{t})=0$, and also when $t_{1}=1, \alpha_{1}(\boldsymbol{t})=f(\boldsymbol{t})$.

Finally, we verify that $\alpha_{i}(\boldsymbol{t}) \leq \frac{t_{i}}{t_{1}} \alpha_{1}(\boldsymbol{t})$. This is again done in a manner similar to Section 4 . Fix values of $\theta_{3}, \ldots, \theta_{m}$, let $T_{\theta_{3}, \ldots, \theta_{n}}\left(t_{1}, \theta_{2}\right)$ be the projection of type space into set of types such that each type $\boldsymbol{t}^{\prime}$ satisfies $t_{1}^{\prime} \geq t_{1}, t_{1}^{\prime} \leq t_{2}^{\prime} \theta$, and $t_{i}^{\prime}=t_{1} \theta_{i}$. Now we can invoke the divergence theorem to conclude that $\alpha_{i}(\boldsymbol{t}) \leq \frac{t_{i}}{t_{1}} \alpha_{1}(\boldsymbol{t})$ if

$$
\frac{d}{d t_{1}}\left(\frac{\int_{y=0}^{t_{1} \theta_{2}} f\left(t_{1}, y, \theta_{3}, \ldots, \theta_{n}\right) \mathrm{d} y}{\int_{y=0}^{t_{1}} f\left(t_{1}, y, \theta_{3}, \ldots, \theta_{n}\right) \mathrm{d} y}\right) \leq 0 .
$$

Notice that fixing $\theta_{3}, \ldots, \theta_{n}$, the above property is exactly what was required in two dimensions, which we showed follows from MR-log-supermodularity. As a result, if the function is MR-logsupermodular in every pair of variables $t_{1}, \theta_{i}$ for $i \geq 2$, then we $\alpha_{i}(\boldsymbol{t}) \leq \frac{t_{i}}{t_{1}} \alpha_{1}(\boldsymbol{t})$. Notice that this property is implied by MR-log-supermodularity of the distribution in all its variables, and therefore is a less demanding condition. We have therefore proved the following lemma.

Lemma 26. If the distribution is MR-log-supermodular in every pair of variables $t_{1}, \theta_{i}$ for $i \geq 2$, then the revenue of an allocation can be upper bounded by

$$
\int_{\boldsymbol{t}} \boldsymbol{x} \cdot\left(1, \frac{t_{2}}{t_{1}}, \ldots, \frac{t_{n}}{t_{1}}\right) \alpha\left(t_{1}\right) f(\boldsymbol{t}) \mathrm{d} \boldsymbol{t}
$$

where

$$
\alpha_{1}(\boldsymbol{t})=t_{1}-\frac{\int_{\boldsymbol{t}^{\prime} \in T\left(t_{1}, 1, \ldots, 1\right)} f\left(\boldsymbol{t}^{\prime}\right) \mathrm{d} \boldsymbol{t}^{\prime}}{\int_{\boldsymbol{t}^{\prime} \in C\left(t_{1}, 1, \ldots, 1\right)} f\left(\boldsymbol{t}^{\prime}\right) \mathrm{d} \boldsymbol{t}^{\prime}} .
$$

\section{B Missing Proofs}

\section{B.1 Proof from Section 4}

Proof of Lemma 14. We need to prove

$$
f^{M R}\left(t_{1}, \theta_{1}\right) \times f^{M R}\left(t_{1}^{\prime}, \theta_{1}^{\prime}\right) \leq f^{M R}\left(t_{1}, \theta_{1}^{\prime}\right) \times f^{M R}\left(t_{1}^{\prime}, \theta_{1}\right), \quad \forall t_{1} \leq t_{1}^{\prime}, \theta_{1} \geq \theta_{1}^{\prime} .
$$

Recall that $f^{M R}\left(t_{1}, \theta_{1}\right)=f\left(t_{1}, t_{1} \theta_{1}\right)$. Since the distribution is a product one, this implies that $f^{M R}\left(t_{1}, \theta_{1}\right)=f_{1}\left(t_{1}\right) f_{2}\left(t_{1} \theta_{1}\right)$. Notice that pair of values $t \theta^{\prime}$ and $t^{\prime} \theta$ have the same geometric mean as the pair $t \theta, t^{\prime} \theta^{\prime}$. Also given the assumptions, $t \theta^{\prime} \leq t \theta, t^{\prime} \theta^{\prime} \leq t^{\prime} \theta$. GG-convexity implies that

$$
f_{2}(t \theta) \times f_{2}\left(t^{\prime} \theta^{\prime}\right) \leq f_{2}\left(t \theta^{\prime}\right) \times f_{2}\left(t^{\prime} \theta\right) .
$$

Multiplying both sides by $f_{1}\left(t_{1}\right) \times f_{1}\left(t_{1}^{\prime}\right)$ we get

$$
f_{1}\left(t_{1}\right) f_{2}(t \theta) \times f_{1}\left(t_{1}^{\prime}\right) f_{2}\left(t^{\prime} \theta^{\prime}\right) \leq f_{1}\left(t_{1}\right) f_{2}\left(t \theta^{\prime}\right) \times f_{1}\left(t_{1}^{\prime}\right) f_{2}\left(t^{\prime} \theta\right),
$$

which since the distribution is a product distribution implies that

$$
f^{M R}\left(t_{1}, \theta_{1}\right) \times f^{M R}\left(t_{1}^{\prime}, \theta_{1}^{\prime}\right) \leq f^{M R}\left(t_{1}, \theta_{1}^{\prime}\right) \times f^{M R}\left(t_{1}^{\prime}, \theta_{1}\right) .
$$




\section{B.2 Proofs from Section 5}

Proof of Lemma 16. Let $\boldsymbol{x}^{p}$ be the allocation corresponding to posting price $p$ for the bundle, that is $x_{1}^{p}(\boldsymbol{t})=x_{2}^{p}(\boldsymbol{t})=1$ if $t_{1}+t_{2} \geq p$, and $x_{1}^{p}(\boldsymbol{t})=x_{2}^{p}(\boldsymbol{t})=0$ otherwise. We will show that the virtual surplus of $\boldsymbol{x}^{p}$ is equal to the revenue of posting price $p, R(p)=p\left(1-F_{\text {sum }}(p)\right)$. The virtual surplus is

$$
\begin{aligned}
\int_{\boldsymbol{t} \in T}\left(\boldsymbol{x}^{p} \cdot \boldsymbol{\phi} f\right)(\boldsymbol{t}) \mathrm{d} \boldsymbol{t} & =\int_{\boldsymbol{t} \in T} \boldsymbol{x}^{p}\left(t_{1}, t_{2}\right) \cdot \overline{\boldsymbol{\phi}}\left(t_{1}, t_{2}\right) f\left(t_{1}, t_{2}\right) \mathrm{d} \boldsymbol{t} \\
& =\int_{\boldsymbol{t} \in T, t_{1}+t_{2} \geq p} \phi_{\text {sum }}\left(t_{1}+t_{2}\right) f\left(t_{1}, t_{2}\right) \mathrm{d} \boldsymbol{t} .
\end{aligned}
$$

For a function $h$ on $T$, define $h^{S R}$ to be its transformation to sum-ratio coordinates, that is

$$
h\left(t_{1}, t_{2}\right)=h^{S R}\left(t_{1}+t_{2}, \frac{t_{2}}{t_{1}}\right)
$$

By performing a change of variables $s=t_{1}+t_{2}$, and $\theta=t_{2} / t_{1}$, the virtual surplus of $\boldsymbol{x}^{p}$ can be written as (the Jacobian of the transformation is $\frac{s}{(1+\theta)^{2}}$ )

$$
\begin{aligned}
\int_{s=p}^{1} \int_{\theta=0}^{1} \phi_{\text {sum }}(s) f^{S R}(s, \theta) \frac{s}{(1+\theta)^{2}} \mathrm{~d} \theta \mathrm{d} s & =\int_{s=p}^{1} \phi_{\text {sum }}(s) \int_{\theta=0}^{1} f^{S R}(s, \theta) \frac{s}{(1+\theta)^{2}} \mathrm{~d} \theta \mathrm{d} s \\
& =\int_{s=p}^{1} \phi_{\text {sum }}(s) f_{\text {sum }}(s) \mathrm{d} s
\end{aligned}
$$

Replacing $\phi_{\text {sum }}$ by its definition,

$$
\begin{aligned}
& =\int_{s \geq p} t_{1} f_{\text {sum }}(s)-\left(1-F_{\text {sum }}(s)\right) \mathrm{d} s \\
& =-\int_{s \geq p} \frac{\mathrm{d}}{\mathrm{d} s}\left(s\left(1-F_{\text {sum }}(s)\right) \mathrm{d} s\right. \\
& =R(p)-R(1)=R(p) .
\end{aligned}
$$

Proof of Lemma 20. We assume that $\phi$ satisfying the requirements of the lemma exists, derive the closed form suggested in the lemma, and then verify that the derived $\phi$ indeed satisfies all the required properties. We apply the divergence theorem to $\boldsymbol{\alpha}$ on the trapezoidal subspace of type space defined by types $\boldsymbol{t}^{\prime}$ with $s \leq t_{1}^{\prime}+t_{2}^{\prime} \leq 1, t_{2}^{\prime} / t_{1}^{\prime} \leq \theta$, and $0 \leq t_{1}^{\prime}, t_{2}^{\prime} \leq 1$ (Figure 3 ). The divergence theorem equates the the integral of the vector field $\boldsymbol{\alpha}$ on the boundary of the subspace to the integral of its divergence within the subspace. As the upper boundary of this trapezoidal subspace has slope $t_{2} / t_{1}$, one term in this equality is the integral of $\boldsymbol{\alpha}\left(\boldsymbol{t}^{\prime}\right)$ with the upward orthogonal vector to $\boldsymbol{t}$. Differentiating this integral gives the desired quantity.

Applying the divergence theorem to $\boldsymbol{\alpha}$ on the trapezoid and expressing the top boundary as the interior divergence minus the other three boundaries gives:

$$
\int_{\boldsymbol{t}^{\prime} \in \mathrm{TOP}(s, \theta)} \boldsymbol{\eta}\left(\boldsymbol{t}^{\prime}\right) \cdot \boldsymbol{\alpha}\left(\boldsymbol{t}^{\prime}\right) \mathrm{d} \boldsymbol{t}^{\prime}=\int_{\boldsymbol{t}^{\prime} \in \operatorname{INTERIOR}(s, \theta)} \nabla \cdot \boldsymbol{\alpha}\left(\boldsymbol{t}^{\prime}\right) \mathrm{d} \boldsymbol{t}^{\prime}-\int_{\boldsymbol{t}^{\prime} \in\{\mathrm{RIGHT}, \text { BOTTOM,LEFT }\}(s, \theta)} \boldsymbol{\eta}\left(\boldsymbol{t}^{\prime}\right) \cdot \boldsymbol{\alpha}\left(\boldsymbol{t}^{\prime}\right) \mathrm{d} \boldsymbol{t}^{\prime} .
$$


Since $\boldsymbol{\alpha} / f$ is a strong amortization of utility, the divergence density equality and boundary orthogonality imply that the integral over the interior simplifies and the integrals over the right and bottom boundary are zero, respectively. We have,

$$
\int_{\boldsymbol{t}^{\prime} \in \mathrm{TOP}(s, \theta)} \boldsymbol{\eta}\left(\boldsymbol{t}^{\prime}\right) \cdot \boldsymbol{\alpha}\left(\boldsymbol{t}^{\prime}\right) \mathrm{d} \boldsymbol{t}^{\prime}=-\int_{\boldsymbol{t}^{\prime} \in \operatorname{INTERIOR}(s, \theta)} f\left(\boldsymbol{t}^{\prime}\right) \mathrm{d} \boldsymbol{t}^{\prime}-\int_{\boldsymbol{t}^{\prime} \in \operatorname{LEFT}(s, \theta)} \boldsymbol{\eta}\left(\boldsymbol{t}^{\prime}\right) \cdot \boldsymbol{\alpha}\left(\boldsymbol{t}^{\prime}\right) \mathrm{d} \boldsymbol{t}^{\prime} .
$$

For the trapezoid parameterized by $(s, \theta)$ these integrals are (recall that the Jacobian of the transformation from $\boldsymbol{t}$ to $(s, \theta)$ is $\left.\frac{s}{(1+\theta)^{2}}\right)$,

$$
\int_{s^{\prime}=s}^{1} \frac{\boldsymbol{\alpha}^{S R}\left(s^{\prime}, \theta\right) \cdot(-\theta, 1)}{1+\theta} \mathrm{d} s^{\prime}=-\int_{s^{\prime}=s}^{1} \int_{\theta^{\prime}=0}^{\theta} \frac{f^{S R}\left(s^{\prime}, \theta^{\prime}\right) \cdot s}{\left(1+\theta^{\prime}\right)^{2}} \mathrm{~d} \theta^{\prime} \mathrm{d} s^{\prime}+\int_{\theta^{\prime}=0}^{\theta} \frac{\boldsymbol{\alpha}^{S R}\left(s, \theta^{\prime}\right) \cdot(-1,-1) s}{\left(1+\theta^{\prime}\right)^{2}} \mathrm{~d} \theta^{\prime} .
$$

Differentiating with respect to $s$ gives,

$$
\frac{\boldsymbol{\alpha}^{S R}(s, \theta) \cdot(-\theta, 1)}{1+\theta}=\int_{\theta^{\prime}=0}^{\theta} \frac{f^{S R}\left(s, \theta^{\prime}\right) \cdot s}{\left(1+\theta^{\prime}\right)^{2}} \mathrm{~d} \theta^{\prime}-\frac{\mathrm{d}}{\mathrm{d} s} \int_{\theta^{\prime}=0}^{\theta} \frac{\boldsymbol{\alpha}^{S R}\left(s, \theta^{\prime}\right) \cdot(-1,-1) s}{\left(1+\theta^{\prime}\right)^{2}} \mathrm{~d} \theta^{\prime} .
$$

On the right-hand side, multiply first term by $\frac{f_{\text {sum }}(s)}{f_{\text {sum }}(s)}=1$. The assumption that $\phi_{1}+\phi_{2}=$ $\phi_{\text {sum }}\left(t_{1}+t_{2}\right)$ implies that $\alpha_{1}^{S R}(s, \theta)+\alpha_{2}^{S R}(s, \theta)=\frac{1-F_{\text {sum }}(s)}{f_{\text {sum }}(s)} f^{S R}(s, \theta)$ for all $s$ and $\theta$. These two terms then simplify by the product rule for differentiation to give the identity of the lemma.

$$
\frac{\boldsymbol{\alpha}^{S R}(s, \theta) \cdot(-\theta, 1)}{1+\theta}=f_{\text {sum }}(s) \frac{\int_{\theta^{\prime}=0}^{\theta} \frac{f^{S R}\left(s, \theta^{\prime}\right) \cdot s}{\left(1+\theta^{\prime}\right)^{2}} \mathrm{~d} \theta^{\prime}}{f_{\text {sum }}(s)}+\frac{\mathrm{d}}{\mathrm{d} s}\left[\left(1-F_{\max }(s)\right) \frac{\int_{\theta^{\prime}=0}^{\theta} \frac{f^{S R}\left(s, \theta^{\prime}\right) \cdot s}{\left(1+\theta^{\prime}\right)^{2}} \mathrm{~d} \theta^{\prime}}{f_{\max }\left(t_{1}\right)}\right] .
$$

By definition and change of variables, $F(s, \theta)=\int_{t: t_{1}+t_{2} \leq s, t_{2} / t_{1} \leq \theta} f(\boldsymbol{t}) \mathrm{d} \boldsymbol{t}=\int_{s^{\prime} \leq s} \int_{\theta^{\prime} \leq \theta} f^{S R}\left(s^{\prime}, \theta^{\prime}\right) \frac{s^{\prime}}{\left(1+\theta^{\prime}\right)^{2}} \mathrm{~d} \theta^{\prime} \mathrm{d} s^{\prime}$. Therefore, $f(s, \theta)=\int_{\theta^{\prime} \leq \theta} f^{S R}\left(s, \theta^{\prime}\right) \frac{s}{\left(1+\theta^{\prime}\right)^{2}} \mathrm{~d} \theta^{\prime}$. Plugging this definition into the above equation, we get

$$
\begin{aligned}
\frac{\boldsymbol{\alpha}^{S R}(s, \theta) \cdot(-\theta, 1)}{1+\theta} & =f_{\text {sum }}(s) \frac{f_{\text {sum }}(s, \theta)}{f_{\text {sum }}(s)}+\frac{\mathrm{d}}{\mathrm{d} s}\left[\left(1-F_{\text {sum }}(s)\right) \frac{f_{\text {sum }}(s, \theta)}{f_{\text {sum }}(s)}\right] \\
& =\left(1-F_{\text {sum }}(s)\right) \frac{\mathrm{d}}{\mathrm{d} s}\left[\frac{f_{\text {sum }}(s, \theta)}{f_{\text {sum }}(s)}\right]
\end{aligned}
$$

As a result,

$$
\boldsymbol{\alpha}^{S R}(s, \theta) \cdot(-\theta, 1)=(1+\theta)\left(1-F_{\text {sum }}(s)\right) \frac{\mathrm{d}}{\mathrm{d} s}\left[\frac{f_{\text {sum }}(s, \theta)}{f_{\text {sum }}(s)}\right] .
$$

We can now use the above equation, together with $\alpha_{1}^{S R}(s, \theta)+\alpha_{2}^{S R}(s, \theta)=\frac{1-F_{\text {sum }}(s)}{f_{\text {sum }}(s)} f^{S R}(s, \theta)$ to solve for $\alpha_{1}$.

Proof of Lemma 21. We prove that for any $\theta, s$, and $s^{\prime}$ such that $s<s^{\prime}$,

$$
\frac{f_{\text {sum }}(s, \theta)}{f_{\text {sum }}(s, 1)} \leq \frac{f_{\text {sum }}\left(s^{\prime}, \theta\right)}{f_{\text {sum }}\left(s^{\prime}, 1\right)}
$$


The proof first converts the above form into max-ratio coordinates, applies SR-log-submodularity, and then transforms back to the standard form. Before applying SR-log-submodularity, we break down the integral set into two set, and apply SR-log-submodularity to only one of the integrals. More particularly, notice that

$$
\begin{aligned}
& f_{\text {sum }}(s, \theta) \times f_{\text {sum }}\left(s^{\prime}, 1\right) \\
& =\int_{\theta^{\prime}=0}^{\theta} f^{S R}\left(s, \theta^{\prime}\right) \frac{s}{\left(1+\theta^{\prime}\right)^{2}} \mathrm{~d} \theta^{\prime} \times \int_{\theta^{\prime \prime}=0}^{1} f^{S R}\left(s^{\prime}, \theta^{\prime \prime}\right) \frac{s^{\prime}}{\left(1+\theta^{\prime \prime}\right)^{2}} \mathrm{~d} \theta^{\prime \prime} \\
& =\int_{\theta^{\prime}=0}^{\theta} \int_{\theta^{\prime \prime}=0}^{\theta} f^{S R}\left(s, \theta^{\prime}\right) \frac{s}{\left(1+\theta^{\prime}\right)^{2}} f^{S R}\left(s^{\prime}, \theta^{\prime \prime}\right) \frac{s^{\prime}}{\left(1+\theta^{\prime \prime}\right)^{2}} \mathrm{~d} \theta^{\prime \prime} \mathrm{d} \theta^{\prime} \\
& +\int_{\theta^{\prime}=0}^{\theta} \int_{\theta^{\prime \prime}=\theta}^{1} f^{S R}\left(s, \theta^{\prime}\right) \frac{s}{\left(1+\theta^{\prime}\right)^{2}} f^{S R}\left(s^{\prime}, \theta^{\prime \prime}\right) \frac{s^{\prime}}{\left(1+\theta^{\prime \prime}\right)^{2}} \mathrm{~d} \theta^{\prime \prime} \mathrm{d} \theta^{\prime} \quad \text { (separate double integral into two sets) } \\
& \geq \int_{\theta^{\prime}=0}^{\theta} \int_{\theta^{\prime \prime}=0}^{\theta} f^{S R}\left(s, \theta^{\prime \prime}\right) \frac{s}{\left(1+\theta^{\prime \prime}\right)^{2}} f^{S R}\left(s^{\prime}, \theta^{\prime}\right) \frac{s^{\prime}}{\left(1+\theta^{\prime}\right)^{2}} \mathrm{~d} \theta^{\prime} \mathrm{d} \theta^{\prime \prime} \\
& +\int_{\theta^{\prime}=0}^{\theta} \int_{\theta^{\prime \prime}=\theta}^{1} f^{S R}\left(s, \theta^{\prime \prime}\right) \frac{s}{\left(1+\theta^{\prime \prime}\right)^{2}} f^{S R}\left(s^{\prime}, \theta^{\prime}\right) \frac{s^{\prime}}{\left(1+\theta^{\prime}\right)^{2}} \mathrm{~d} \theta^{\prime \prime} \mathrm{d} \theta^{\prime} \quad \text { (apply MR-log-supermodularity) } \\
& =\int_{\theta^{\prime \prime}=0}^{1} f^{S R}\left(s, \theta^{\prime \prime}\right) \frac{s}{\left(1+\theta^{\prime \prime}\right)^{2}} \mathrm{~d} \theta^{\prime \prime} \int_{\theta^{\prime}=0}^{\theta} f^{S R}\left(s^{\prime}, \theta^{\prime}\right) \frac{s^{\prime}}{\left(1+\theta^{\prime}\right)^{2}} \mathrm{~d} \theta^{\prime} \\
& =f_{\text {sum }}(s, 1) \times f_{\text {sum }}\left(s^{\prime}, \theta^{\prime}\right) \text {. }
\end{aligned}
$$

\section{B.3 Proofs from Section 6.3}

Proof of Lemma 24. Since $\phi_{2}(\boldsymbol{t}) \leq \frac{t_{2}}{t_{1}} \phi_{1}(\boldsymbol{t})$, the revenue of an allocation function can be upper bounded as follows,

$$
\iint \boldsymbol{x}(\boldsymbol{t}) \cdot\left(\phi_{1}(\boldsymbol{t}), \phi_{2}(\boldsymbol{t})\right) f(\boldsymbol{t}) \mathrm{d}(\boldsymbol{t}) \leq \iint \boldsymbol{x}(\boldsymbol{t}) \cdot\left(1, \frac{t_{2}}{t_{1}}\right) \phi_{1}(\boldsymbol{t}) f(\boldsymbol{t}) \mathrm{d} \boldsymbol{t}
$$

After a changing variables according to $\theta=\frac{t_{2}}{t_{1}}$, the upper bound becomes

$$
\int_{t_{1}} \int_{\theta=0}^{1} \boldsymbol{x}^{M R}\left(t_{1}, \theta\right) \cdot(1, \theta) \phi^{M R}\left(t_{1}, \theta\right) f^{M R}\left(t_{1}, \theta\right) t_{1} \mathrm{~d} \theta \mathrm{d} t_{1},
$$

where $\phi^{M R}\left(t_{1}, \theta\right)=\phi\left(t_{1}, t_{1} \theta\right)$. For a function $h$ on $T$, define $h^{Q R}$ to be its transformation to quantile-ratio coordinates, that is

$$
h\left(t_{1}, t_{2}\right)=h^{Q R}\left(\int_{T=T\left(t_{1}, 1\right)} f(\boldsymbol{t}) \mathrm{d} \boldsymbol{t}, \frac{t_{2}}{t_{1}}\right)
$$

We perform another change of variables according to $q=\int_{T=T\left(t_{1}, 1\right)} f(\boldsymbol{t}) \mathrm{d} \boldsymbol{t}$, and rewrite the upper bound as

$$
\int_{q} \int_{\theta} \boldsymbol{x}^{Q R}(q, \theta) \cdot(1, \theta) \phi^{Q R}(q) \mathrm{d} \theta \mathrm{d} q
$$


Using integration by parts,

$$
\int_{q} \int_{\theta} \boldsymbol{x}^{Q R}(q, \theta) \cdot(1, \theta) \boldsymbol{\phi}^{Q R}(q) \mathrm{d} \theta \mathrm{d} q=\int_{q} \int_{\theta} \frac{d}{d q} \boldsymbol{x}^{Q R}(q, \theta) \cdot(1, \theta) R(q) \mathrm{d} \theta \mathrm{d} q
$$

where $R(q)$ is the revenue of posting a price that is accepted with probability $q$. Now let $\bar{R}$ be the convex hull of function $R$. In particular, $\bar{R}^{\prime}(q)$ is non-decreasing and $\bar{R}(q) \geq R(q)$. We next show that $\frac{d}{d q} \boldsymbol{x}^{Q R}(q, \theta) \cdot(1, \theta) \geq 0$ for any incentive compatible allocation rule.

Recall that the incentive compatibility condition states that, for all $\boldsymbol{t}, \boldsymbol{t}^{\prime}$,

$$
\left(\boldsymbol{x}\left(\boldsymbol{t}^{\prime}\right)-\boldsymbol{x}(\boldsymbol{t})\right) \cdot\left(\boldsymbol{t}^{\prime}-\boldsymbol{t}\right) \geq 0
$$

Setting $\boldsymbol{t}^{\prime}=\boldsymbol{t}(1+\epsilon)$, this becomes

$$
(\boldsymbol{x}(\boldsymbol{t}(1+\epsilon))-\boldsymbol{x}(\boldsymbol{t})) \cdot(\boldsymbol{t} \epsilon) \geq 0 .
$$

When $\epsilon$ goes to zero, this give us the following property

$$
\frac{d}{d t_{1}} \boldsymbol{x}(\boldsymbol{t}) \cdot \boldsymbol{t} \geq 0
$$

Now notice that $q=\int_{T=T(t, 1)} f(\boldsymbol{t}) \mathrm{d} \boldsymbol{t}$ is a monotone function of $t_{1}$, which implies the desired property

$$
\frac{d}{d q} \boldsymbol{x}^{Q R}(q, \theta) \cdot(1, \theta) \geq 0
$$

The facts that $\frac{d}{d q} \boldsymbol{x}^{Q R}(q, \theta) \cdot(1, \theta) \geq 0$ and $\bar{R}(q) \geq R(q)$ imply that we can upper bound revenue as follows

$$
\int_{q} \int_{\theta} \frac{d}{d q} \boldsymbol{x}^{Q R}(q, \theta)(1, \theta) R(q) \mathrm{d} \theta \mathrm{d} q \leq \int_{q} \int_{\theta} \frac{d}{d q} \boldsymbol{x}^{Q R}(q, \theta)(1, \theta) \bar{R}(q) \mathrm{d} \theta \mathrm{d} q
$$

We now study the allocation rule that optimizes virtual value function $(1, \theta) R^{\prime}(q)$. Notice that when $\bar{R}(q)>R(q)$, we have that $\bar{R}^{\prime}(q)$ is a constant and therefore $\frac{d}{d q} \boldsymbol{x}^{Q R}=0$. This implies that $\hat{R}^{\prime}(q)$ can be used as an upper bound on revenue, and for the expected virtual value of the optimal allocation exactly equals revenue. 